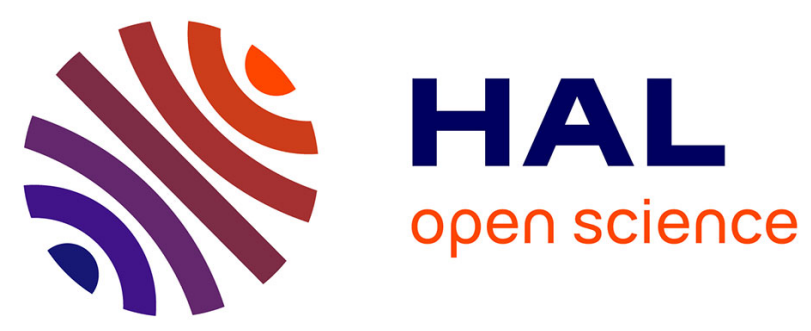

\title{
Phase-field modeling with the TAF-ID of incipient melting and oxygen transport in nuclear fuel during power transients
}

Clément Introïni, Jérôme Sercombe, Isabelle Ramière, Romain Le Tellier

\section{To cite this version:}

Clément Introïni, Jérôme Sercombe, Isabelle Ramière, Romain Le Tellier. Phase-field modeling with the TAF-ID of incipient melting and oxygen transport in nuclear fuel during power transients. Journal of Nuclear Materials, 2021, 556, pp.153173. 10.1016/j.jnucmat.2021.153173 . cea-03581220

\section{HAL Id: cea-03581220 \\ https://hal-cea.archives-ouvertes.fr/cea-03581220}

Submitted on 19 Feb 2022

HAL is a multi-disciplinary open access archive for the deposit and dissemination of scientific research documents, whether they are published or not. The documents may come from teaching and research institutions in France or abroad, or from public or private research centers.
L'archive ouverte pluridisciplinaire HAL, est destinée au dépôt et à la diffusion de documents scientifiques de niveau recherche, publiés ou non, émanant des établissements d'enseignement et de recherche français ou étrangers, des laboratoires publics ou privés. 


\title{
Phase-field modeling with the TAF-ID of incipient melting and oxygen transport in nuclear fuel during power transients
}

\author{
C. Introïni, J. Sercombe, I. Ramière ${ }^{a}$, R. Le Tellier ${ }^{b}$ \\ ${ }^{a}$ CEA, DES, IRESNE, DEC, Cadarache F-13108 Saint-Paul-Lez-Durance, France \\ ${ }^{b}$ CEA, DES, IRESNE, DTN, Cadarache F-13108 Saint-Paul-Lez-Durance, France
}

\begin{abstract}
In this paper, a phase-field model is developed for a two phase compositional (multicomponent) system and combined with the thermodynamic description provided by a CALPHAD database. The model is applied to a uranium-oxygen binary system within a solid/liquid mixture to simulate incipient melting and oxygen transport (oxygen-uranium inter-diffusion and Soret effect) in the fuel with thermodynamic properties coming from the Thermodynamics for Advanced Fuels-International Database (TAF-ID). The interface with the TAF-ID required the coupling of the phase-field model with the OpenCalphad thermochemical solver. This coupling led to the development of a partitioned scheme that is solved at each time step with a fixed-point algorithm. To analyze the response of and to parameterize the phase-field model, two 1D demonstration problems from the open literature have been considered and solved using a finite-difference scheme. The first problem deals with oxygen transport in $\mathrm{UO}_{2.005}$ fuel under an imposed parabolic temperature profile below liquidus. The second one describes the incipient melting and oxygen transport in oxidized fuel under an increasing temperature profile. Oxygen thermal diffusion is considered both in the solid and liquid phases. All the results presented in this paper are in good agreement with those already published but are here performed considering a direct coupling with the TAF-ID database. They demonstrate the thermodynamic consistency of the phase-field model as well as its capability to simulate incipient melting and oxygen transport in the fuel with advanced thermodynamics databases.
\end{abstract}

Keywords: phase-field, oxygen transport, incipient fuel melting, CALPHAD, TAF-ID, PLEIADES, OPENCALPHAD

\section{Introduction}

In nuclear reactors, the fuel experiences high temperatures and large temperature gradients. During extreme power transients, partial fuel core melting might occur. Modeling the phase change is complex due to the Fission Products (FPs) generated in the fuel material that modifies the local equilibria. In case of defective fuels, transport 5 mechanisms such as oxygen thermal diffusion in the solid phase might also be involved [1]. Since the fuel phase change depends on the local oxygen-to-uranium ratio [2], a strong coupling between the heat flux, the oxygen redistribution process and the local chemical equilibria in the fuel must be considered. In the last decade, the thermochemical state of the FPs and their migration in the fuel have received greater attention in conditions far from fuel melting [3, 4, 5]. Thermochemical solvers capable of handling large and complex chemical systems have been developed [6, 7, 8, 9]. Thanks to modern interface softwares, they have been incorporated in fuel performance codes [4, 5, 10, 11, 12, 13]. To our knowledge, there is no fuel performance code that is nowadays capable of handling simultaneously fuel melting, oxygen thermal diffusion and local chemical equilibria in the fuel.

The coupling between thermal diffusion, oxygen thermal diffusion and local chemical equilibria is already available in the PLEIADES/ALCYONE 2.1 fuel performance code [4, 5, 13 but limited to temperatures below the liquidus and to the constant power plateau of power ramps. Indeed, it relies on the thermodynamic properties of the TBASE database [14] that does not contain any description of the liquid phase diagram. The thermal diffusion problem is furthermore formulated as a function of the fuel stoichiometry deviation. Recently, Simunovic \& al. [11] have developed a model for oxygen transport in LWR fuel that has been performed in the BISON fuel performance code [15, 16]. The model is formulated such that it can be combined with CALPHAD databases [2, 17, 18. 
In this approach, the driving force for the oxygen thermal diffusion mechanism is the oxygen chemical potential gradient and not the stoichiometry deviation gradient in the fuel. This formulation facilitates the handling of multi-component systems such as those encountered in irradiated fuels. Moreover, the Soret effect is formally neglected assuming that the gradient of the oxygen chemical potential accounts for the effects of both composition and temperature gradients.

The modeling actually provided by the BISON or PLEIADES/ALCYONE fuel performance codes is however limited to temperatures below the liquidus. To overcome this limit, phase-field modeling appears as an interesting method since it is an efficient numerical approach for mesoscopic modeling of complex micro-structures [19]. As shown in the open literature, phase-field methods are particularly suitable for physical systems with complex topology changes such as, multiphase multicomponent flows [20, 21, 22, 23] and brittle fracture [24]. More interesting for nuclear fuel applications, solute diffusion in a binary alloy [25], liquid phase segregation in the U-O binary system [26, solidification in binary alloys [27, 28], sub-oxidized corium plane front solidification [29], constituent redistribution in U-Zr fuels [30, 31] and incipient melting in defective fuel [32, 33, 34] have all been successfully treated with a phase-field modeling approach. Contrary to sharp interfaces models in which the interfaces are viewed as discontinuous surfaces with excess physical properties, phase-field modeling considers the interfaces as thin regions of finite thickness across which variables vary continuously and smoothly. This is why phase-field methods belong to the family of diffuse interface methods. With such a diffuse description of the interfaces, phase-field modeling enables an implicit tracking of the interfaces thanks to intensive phase-field variables, also called order parameters, that are constant in each bulk phase and vary smoothly across the interfaces.

Another great interest of phase-field modeling is the possible and natural coupling with a thermodynamic

40 CALPHAD database from which thermodynamic fluxes involved in the governing equations and diffusion kinetics parameters such as mobilities or self-diffusion coefficients can be directly calculated [35 36. As previously mentioned, most of the studies conducted in the PLEIADES platform about nuclear fuel thermochemistry rely on a TBASE database. Recently, the Thermodynamics for Advanced Fuels-International Database (TAF-ID) 2 [37 38 39] has been incorporated in the PLEIADES platform [12, 40]. Based on the CALPHAD method and the

45 Compound Energy Formalism (CEF) [41], the TAF-ID thermodynamic models take crystal and defect structure of the phases into account, such as, for example, sub-lattices, interstitials or vacancies. Moreover, compared to the TBASE, the TAF-ID provides a more complex description of irradiated fuel and enables to describe the liquid solution phase using an ionic model [42].

In the open literature, different approaches are used to couple a phase-field model with a CALPHAD database.

so When the variables of the CALPHAD phase Gibbs energy function are the same than those of the phase-field model, a straightforward approach is to use directly an analytical expression of the Gibbs energy function (see [30, 31, 32, 33, 34]). However, in complex thermodynamic systems with advanced thermodynamic representations, this is no longer possible as the homogeneous energy function of the phase-field model cannot be directly identified with a CALPHAD phase Gibbs energy function. Additional modeling hypotheses are needed. In most 55 cases, a "local» energy minimization is involved (e.g. local equilibrium of redox mechanisms in [26]) and the interfacing of a CALPHAD thermodynamic description with a phase-field becomes a computational issue to be handled with care [43]. The use of a thermodynamic solver (Gibbs energy minimizer) provides in fact an exact and continuous representation of the composition dependence of the CALPHAD Gibbs energies. The difficulties that can be encountered are an extra computational cost and the management of non convergence problems when so coupled to the phase-field model. In the present work, the experience acquired from the implementation of a thermochemical solver in the fuel performance code ALCYONE led to choose this type of direct coupling with a thermodynamic solver.

In this work, the coupling between thermal diffusion, oxygen thermal diffusion and local chemical equilibria available in the PLEIADES platform is expanded to temperatures above the liquidus. A new phase-field model

65 for a two-phase compositional (multicomponent) system is proposed. The general formulation of the governing equations is first derived in the frame of the Thermodynamics of Irreversible Processes (TIP) [44] by using suitable thermodynamic closures. More particularly, the description of the liquid-solid interface is based on the Kim-KimSuzuki model [28. A reduction of this general model to a uranium-oxygen binary system in a solid/liquid mixture is then detailed. Its implementation is explained with a focus on the numerical scheme that efficiently couples the phase-field model with the OpenCalphad thermo-chemical solver [9] [13]. Finally, to assess the efficiency of the coupling, two demonstration problems dealing with oxygen thermal diffusion and incipient fuel melting are 
presented. For this purpose, the OpenCalphad calculations are performed with the thermodynamic data coming from the TAF-ID [2].

\section{Thermodynamically consistent derivation of a phase-field model for a two-phase compositional system}

75 with the thermodynamic description of the targeted physical system and, more particularly, with the definition of the thermodynamic closures. Then, we derive the governing equations in the frame of the TIP [44].

Before describing the thermodynamic system, it is noteworthy mentioning that the hydrodynamics and structural mechanics effects such as, respectively, convection and fuel cracking, are fully neglected in this work.

\subsection{Statement of the problem and thermodynamic closures}

We begin by introducing a non-conserved phase-field variable $\varphi$ to simulate the two-phase transformation [19]. In this context, $\varphi$ is often called order parameter as in 45], to cite among others. But here, the phase-field variable rather plays the role of an indicator function. That is why, in the following, $\varphi$ is referred to as phaseindicator. It is equal to one in the solid phase, zero in the liquid phase and varies continuously and smoothly between zero and one across the liquid-solid interface:

$$
\begin{aligned}
& \varphi=1 \quad \text { (solid phase) } \\
& \varphi=0 \text { (liquid phase) } \\
& \varphi \in] 0,1[\text { (liquid-solid interface) }
\end{aligned}
$$

Then, to describe the compositional system, we define a set of conserved variables which must fulfill the following constraint:

$$
\sum_{i=1}^{n} x_{i}=1
$$

where $n$ is an arbitrary finite number of components. Here-above, the $x_{i}$ variable refers to the molar fraction of the $i$ th componen 1 . As the gaseous phase is not described, we assume that each component is incompressible. The thermal expansion and the volume change upon phase transition are also neglected. In these conditions, the molar density $\left(\mathrm{mol} . \mathrm{m}^{-3}\right)$ of the liquid-solid mixture $\rho$ or, equivalently, the molar volume $V_{m}=1 / \rho$ only depends n the composition of the system but, here, it is assumed to be constant for simplicity.

At this stage, for the sake of clarity, let us define some notations that will be used in this section: the subscript ${ }_{i}$ denotes a quantity related to the $i$ th component; the superscripts ${ }^{l}$ and ${ }^{s}$ refer to the liquid and solid phases, respectively; and the curly bracket $\{\cdot\}$ defines a set of variables indexed by the number of components.

In this work, we consider that a thermodynamic description of the two-phase compositional system by the molar Gibbs energy $g\left(\mathrm{~J} \cdot \mathrm{mol}^{-1}\right)$ is valid. In the case of a diffuse interface modeling, $g$ depends not only on the pressure $p$, the temperature $T$, the phase-field variable $\varphi$ and the molar fractions $\left\{x_{i}\right\}$ but also on the gradients $\nabla \varphi$ and $\left\{\nabla x_{i}\right\}$. Under these circumstances, the proposed closure for the Gibbs energy density function reads:

$$
\rho g\left(p, T,\left\{x_{i}\right\}, \varphi,\left\{\nabla x_{i}\right\}, \nabla \varphi\right)=\rho g_{0}\left(p, T,\left\{x_{i}\right\}, \varphi\right)+W(\varphi)+\sum_{i=1}^{n} \frac{\lambda_{i}}{2}\left|\nabla x_{i}\right|^{2}+\frac{\kappa}{2}|\nabla \varphi|^{2}
$$

where $\lambda_{i}$ and $\kappa$ are gradient energy coefficients $\left(\mathrm{J}_{\mathrm{m}} \mathrm{m}^{-1}\right)$, here assumed to be constant. In Eq. (3), $g_{0}\left(p, T,\left\{x_{i}\right\}, \varphi\right)$ is a molar homogeneous free energy $\left(\mathrm{J}_{\mathrm{mol}} \mathrm{mol}^{-1}\right)$ and $W(\varphi)$ corresponds to the well-known double-well potential. The latter is given by:

$$
W(\varphi)=\omega \varphi^{2}(1-\varphi)^{2}
$$

where $\omega$ is the height of the double-well $\left(\mathrm{J}_{\mathrm{m}} \mathrm{m}^{-3}\right)$. This parameter will be defined later. A schematic representation

\footnotetext{
${ }^{1}$ Using the terminology considered in [8], it is important to keep in mind that the derivation of the model is based on the mole fractions of components, not on the mole fractions of species nor on the sites fractions as in 46
} 
shown. These graphs show that the double-well potential has two local minima in $\varphi=0$ and $\varphi=1$ that correspond to the pure phases. It acts to separate the two phases contrary to gradient energy terms that can be viewed as a regularization of the interfaces. It is the competition between these two terms that contributes to have a diffuse interface.

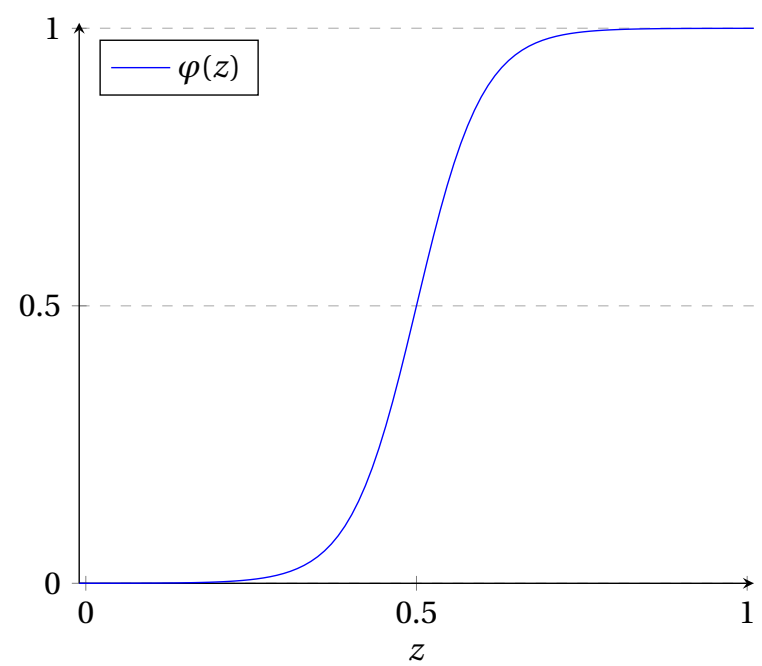

(a) Phase-indicator profile

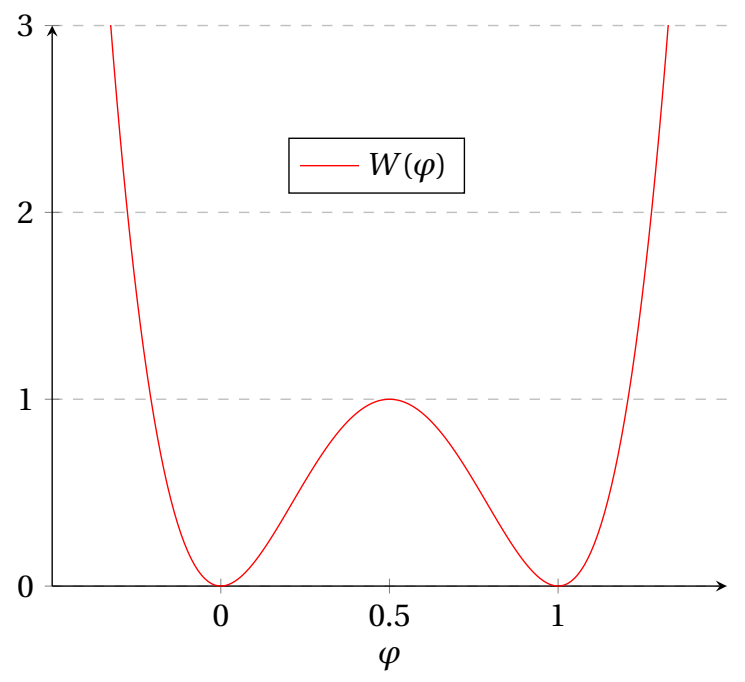

(c) Double-well potential

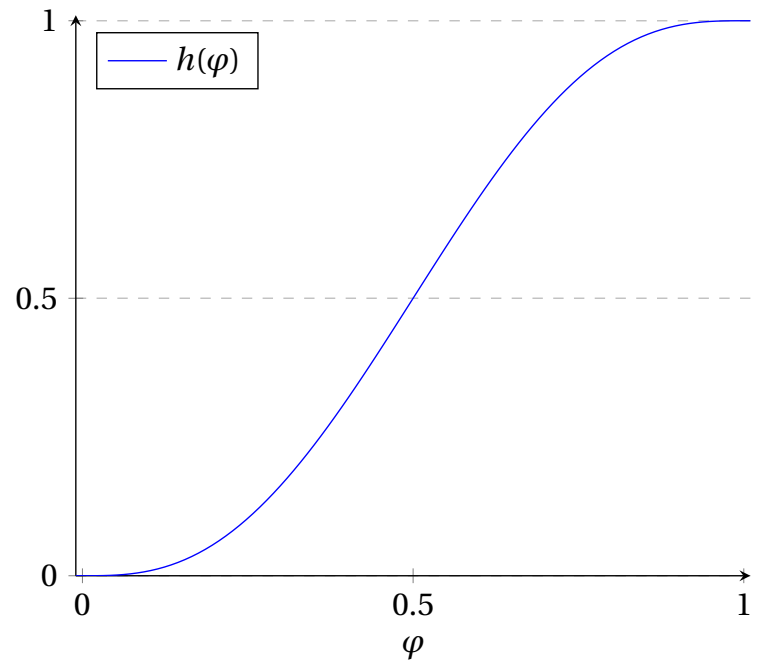

(b) Interpolation function

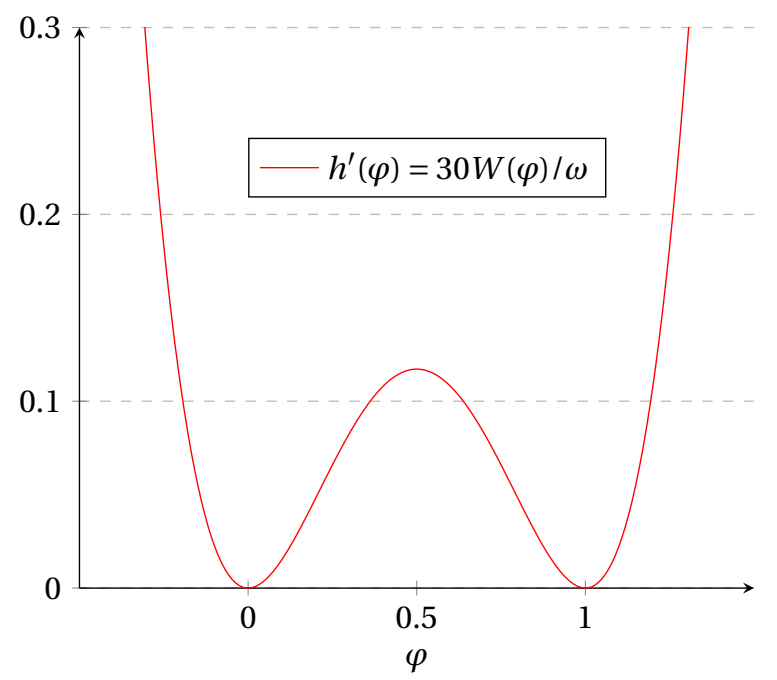

(d) Derivative of the interpolation function

Figure 1: Schematic representation of the smooth variation of the phase-indicator $\varphi$ across the interface, the double-well potential $W(\varphi)$, the interpolation function $h(\varphi)$ and its derivative $h^{\prime}(\varphi)$

At this stage, it remains to express $g_{0}$ in Eq. 3. Here, we assume that the molar homogeneous free energy is defined by the weighting of pure molar Gibbs energies $g^{s}$ and $g^{l}$ associated to the solid and liquid phases, respectively. This reads:

$$
g_{0}\left(p, T,\left\{x_{i}\right\}, \varphi\right)=h(\varphi) g^{s}\left(p, T, x_{i}^{s}\right)+[1-h(\varphi)] g^{l}\left(p, T, x_{i}^{l}\right)
$$

where $h(\varphi)$ is an interpolation function. Here, according to [19], we consider the following expression:

$$
h(\varphi)=\varphi^{3}\left(6 \varphi^{2}-15 \varphi+10\right)
$$


whose first derivative with respect to $\varphi$ is proportional to the double-well potential. We will see later that $h^{\prime}(\varphi)$ appears in the phase-field model. The function $h(\varphi)$ and its derivative $h^{\prime}(\varphi)$ are schematically presented in Fig 1 .

Another important thermodynamic closure is the description of the interfacial region. Following [29, 30, 32], we consider the well-known Kim-Kim-Suzuki model [28] to describe the interface as a mixture of solid and liquid phases, with different composition but with the same chemical potentials.

$$
\begin{aligned}
x_{i} & =h(\varphi) x_{i}^{s}+[1-h(\varphi)] x_{i}^{l} \\
\frac{\partial g^{s}}{\partial x_{i}^{s}}\left(x_{i}^{s}\right) & =\frac{\partial g^{l}}{\partial x_{i}^{l}}\left(x_{i}^{l}\right)
\end{aligned}
$$

Note that $\partial g / \partial x_{i}$ differs from the standard definition of the chemical potential but is used here since the change in the total density $\rho$ induced by the components' diffusion is neglected. For more details about the strength of such a model compared to other approaches [27], we refer the interested reader to [19, 28, 46] and the references therein. The thermodynamic closures given by Eq. (3) to Eqs. (7) are similar to those proposed in [46]. They will be used in the next section to derive the governing equations.

\subsection{Governing equations}

In this section, we derive the governing equations used to describe a two-phase compositional system. This is done from the thermodynamic closures proposed in \$2.1 in the frame of the TIP [44].

To begin, let us consider the following set of governing equations:

$$
\begin{aligned}
\frac{\partial \varphi}{\partial t} & =-J_{\varphi} \\
\frac{\partial \rho x_{i}}{\partial t} & =-\nabla \cdot \mathbf{j}_{i} \quad i=1, \ldots, n \\
\frac{\partial \rho e_{t}}{\partial t} & =-\nabla \cdot \mathbf{q}_{e_{t}} \\
\frac{\partial \rho s}{\partial t} & =-\nabla \cdot \mathbf{q}_{s}+\mathscr{S}
\end{aligned}
$$

where $\mathrm{s}$ is the molar entropy, $\mathscr{S}$ the entropy source, $\mathbf{q}_{s}$ the entropy flux, $e_{t}$ the molar total energy, $\mathbf{q}_{e_{t}}$ the total energy flux, $\mathbf{j}_{i}$ the compositional flux associated to the $i$ th component and $J_{\varphi}$ a flow related to the phase change. In this work, as done in [33], the momentum balance equation is ignored since neither structural mechanics nor hydrodynamics effects are considered. Therefore, the molar total energy $e_{t}$ corresponds to the molar internal energy of the system $e$.

In Eqs. 8], $J_{\varphi}, \mathbf{j}_{i}, \mathbf{q}_{e_{t}}$ and $\mathbf{q}_{s}$ are thermodynamics flow and fluxes that must be specified such that the second law of the thermodynamic holds, namely:

$$
\mathscr{S} \geq 0
$$

For this purpose, the governing equations Eqs. (8) must be combined under the constraint Eq. 2) to express the entropy source $\mathscr{S}$ as a linear combination of thermodynamics forces and fluxes.

As already mentioned, the molar Gibbs energy depends on the pressure $p$, the temperature $T$, the phase-field variable $\varphi$, the molar fractions $\left\{x_{i}\right\}$ but also on the gradients $\nabla \varphi$ and $\left\{\nabla x_{i}\right\}$. Therefore, its differential reads:

$$
d g=-s d T+V_{m} d p+\sum_{i=1}^{n} \mu_{i} d x_{i}+\sum_{i=1}^{n} \boldsymbol{\psi}_{i} \cdot d \nabla x_{i}+\theta d \varphi+\zeta \cdot d \nabla \varphi
$$


where the molar volume $V_{m}$, the molar entropy $s$ and the potentials $\mu_{i}, \boldsymbol{\psi}_{i}, \theta, \zeta$ are defined by:

$$
\begin{aligned}
V_{m} & =\left(\frac{\partial g}{\partial p}\right)_{T, \varphi, \nabla \varphi,\left\{x_{i}\right\},\left\{\nabla x_{i}\right\}} \\
s & =-\left(\frac{\partial g}{\partial T}\right)_{p, \varphi, \nabla \varphi,\left\{x_{i}\right\},\left\{\nabla x_{i}\right\}} \\
\mu_{i} & =\left(\frac{\partial g}{\partial x_{i}}\right)_{p, T, \varphi, \nabla \varphi,\left\{x_{j}\right\}_{j \neq i},\left\{\nabla x_{i}\right\}} \\
\boldsymbol{\psi}_{i} & =\left(\frac{\partial g}{\partial \nabla x_{i}}\right)_{p, T, \varphi,\left\{x_{i}\right\},\left\{\nabla x_{j}\right\}_{j \neq i}} \\
\theta & =\left(\frac{\partial g}{\partial \varphi}\right)_{p, T, \nabla \varphi,\left\{x_{i}\right\},\left\{\nabla x_{i}\right\}} \\
\zeta & =\left(\frac{\partial g}{\partial \nabla \varphi}\right)_{p, T, \varphi,\left\{x_{i}\right\},\left\{\nabla x_{i}\right\}}
\end{aligned}
$$

Now, we define the molar internal energy $e$ as a function of the molar Gibbs energy $g$ by applying the Legendre transformation. This yields to:

$$
e=g-T\left(\frac{\partial g}{\partial T}\right)_{p, \varphi, \nabla \varphi,\left\{x_{i}\right\},\left\{\nabla x_{i}\right\}}-p\left(\frac{\partial g}{\partial p}\right)_{T, \varphi, \nabla \varphi,\left\{x_{i}\right\},\left\{\nabla x_{i}\right\}}
$$

In the following, the subscripts referring to the constant quantities in the partial derivatives will be omitted in order to simplify the notations. The use of Eqs.11] together with Eq.(12) leads to:

$$
e=g+T s-p V_{m}
$$

Now, as the evolution of the two phase compositional system is driven by the minimization of the molar Gibbs energy $g$, the Lagrange multiplier $\beta$ is introduced to ensure the constraint Eq.22, yielding:

$$
e=g+T s-p V_{m}+\beta \sum_{i=1}^{n}\left(x_{i}-1\right)
$$

or, equivalently, the following differential form (where the constant molar volume assumption is applied):

$$
d e=d g+T d s+s d T-V_{m} d p+\beta \sum_{i=1}^{n} d x_{i}
$$

Then, substituting Eq.(10) in Eq.(15) and after performing some straightforward calculations under local thermodynamic equilibrium conditions [44, it yields to:

$$
\frac{\partial e}{\partial t}=T \frac{\partial s}{\partial t}+\sum_{i=1}^{n}\left(\tilde{\mu}_{i}+\beta\right) \frac{\partial x_{i}}{\partial t}+\sum_{i=1}^{n} \nabla \cdot\left(\boldsymbol{\psi}_{i} \frac{\partial x_{i}}{\partial t}\right)+\eta \frac{\partial \varphi}{\partial t}+\nabla \cdot\left(\zeta \frac{\partial \varphi}{\partial t}\right)
$$

where $\tilde{\mu}_{i}$ and $\eta$ are two generalized potentials defined by:

$$
\begin{aligned}
\tilde{\mu}_{i} & =\mu_{i}-\nabla \cdot \boldsymbol{\psi}_{i} \quad i=1, \ldots, n \\
\eta & =\theta-\nabla \cdot \zeta
\end{aligned}
$$

By using Eq. (3) and Eqs. (11), they can be written as:

$$
\begin{aligned}
\tilde{\mu}_{i} & =\mu_{i}-\frac{\lambda_{i}}{\rho} \nabla^{2} x_{i} \quad i=1, \ldots, n \\
\eta & =\frac{\partial g}{\partial \varphi}-\frac{\kappa}{\rho} \nabla^{2} \varphi
\end{aligned}
$$


Finally, the governing equations Eqs. [8] are used to simplify Eq. 16], yielding:

$$
\frac{\partial \rho s}{\partial t}=-\nabla \cdot\left(\frac{1}{T} \mathbf{q}^{\prime}\right)-\frac{1}{T^{2}} \mathbf{q}^{\prime} \cdot \nabla T-\frac{1}{T} \sum_{i=1}^{n} \nabla\left(\tilde{\mu}_{i}+\beta\right) \cdot \mathbf{j}_{i}+\frac{1}{T} \rho \eta J_{\varphi}
$$

where $\mathbf{q}^{\prime}$ is a heat flux given by:

$$
\mathbf{q}^{\prime}=\rho \zeta \frac{\partial \varphi}{\partial t}-\sum_{i=1}^{n}\left[\left(\tilde{\mu}_{i}+\beta\right) \mathbf{j}_{i}+\boldsymbol{\psi}_{i} \frac{\partial\left(\rho x_{i}\right)}{\partial t}\right]+\mathbf{q}_{e_{t}}
$$

From the comparison of Eq. [19] to the entropy balance Eq. $8 d$, one can write:

$$
\begin{aligned}
\mathbf{q}^{\prime} & =T \mathbf{q}_{s} \\
\mathscr{S} & =-\frac{1}{T} \sum_{i=1}^{n} \nabla\left(\tilde{\mu}_{i}+\beta\right) \cdot \mathbf{j}_{i}+\frac{1}{T} \rho \eta J_{\varphi}-\frac{1}{T^{2}} \mathbf{q}^{\prime} \cdot \nabla T
\end{aligned}
$$

As expected, the entropy source given by Eq.22 is now expressed as a linear combination of the thermodynamic forces $\left(\left[\nabla\left(\tilde{\mu}_{i}+\beta\right)\right] / T, \nabla T / T^{2}, \rho \eta / T\right)$ and of the thermodynamic fluxes $\left(-\mathbf{j}_{i},-\mathbf{q}^{\prime}, J_{\varphi}\right)$ responsible for the irreversible processes [4].

In the frame of the TIP, the thermodynamic fluxes may be linked to thermodynamic forces thanks to linear phenomenological relationships 44 47. Before expressing these relationships, let us discuss about the formulation of the entropy source term. Indeed, it is noteworthy to mention that the conjugate pairs $\left(-\mathbf{j}_{i},\left[\nabla\left(\tilde{\mu}_{i}+\beta\right)\right] / T\right)$ and $\left(-\mathbf{q}^{\prime}, \nabla T / T^{2}\right)$ depend directly on how the heat flux is defined in Eq. 20. Another definition of the heat flux will result in a different formulation of the entropy source term with different conjugate pairs. For example, following [11, 44, 48, 49 to cite among others, $\mathscr{S}$ can be formulated as:

$$
\mathscr{S}=-\sum_{i=1}^{n} \nabla\left(\frac{\tilde{\mu}_{i}+\beta}{T}\right) \cdot \mathbf{j}_{i}+\frac{1}{T} \rho \eta J_{\varphi}-\frac{1}{T^{2}} \mathbf{q} \cdot \nabla T
$$

where $\mathbf{q}$ is another heat flux related to $\mathbf{q}_{s}(c f$. Eq.21]) according to:

$$
\mathbf{q}=T \mathbf{q}_{s}+\sum_{i=1}^{n}\left(\tilde{\mu}_{i}+\beta\right) \mathbf{j}_{i}
$$

Compared to Eq.22], the diffusion flux $-\mathbf{j}_{i}$ in Eq. 23] is conjugate to $\nabla\left[\left(\tilde{\mu}_{i}+\beta\right) / T\right]$ instead of $\left[\nabla\left(\tilde{\mu}_{i}+\beta\right)\right] / T$. From Eq. 24, another heat flux $\overline{\mathbf{q}}$ can be defined. It reads:

$$
\overline{\mathbf{q}}=\mathbf{q}-\sum_{i=1}^{n}\left(\frac{\partial h}{\partial x_{i}}-\boldsymbol{\psi}_{i}\right) \mathbf{j}_{i}
$$

where $h=g-T s$ is the molar enthalpy $\left(\mathrm{J} \cdot \mathrm{mol}^{-1}\right.$ ). In Eq. 25), $\mathbf{q}$ can be viewed as a « reduced $»$ heat flux that includes the pure heat conduction but excludes the heat transfer by diffusion [48]. Such a definition of the heat flux leads to the following entropy source term:

$$
\mathscr{S}=-\frac{1}{T} \sum_{i=1}^{n} \nabla_{T}\left(\tilde{\mu}_{i}+\beta\right) \cdot \mathbf{j}_{i}+\frac{1}{T} \rho \eta J_{\varphi}-\frac{1}{T^{2}} \overline{\mathbf{q}} \cdot \nabla T
$$

In Eq.26, the conjugate pairs are different from those obtained in Eq.22) or in Eq. 23. Here, the diffusion flux $-\mathbf{j}_{i}$ is conjugate to $\left[\nabla_{T}\left(\tilde{\mu}_{i}+\beta\right)\right] / T$ where $\nabla_{T}$ is the gradient taken at constant temperature. It means that the thermodynamic force $\left[\nabla_{T}\left(\tilde{\mu}_{i}+\beta\right)\right] / T$ does not contain any temperature gradient contribution, contrary to the thermodynamic force $\left[\nabla\left(\tilde{\mu}_{i}+\beta\right)\right] / T$ that results from the heat flux $\mathbf{q}^{\prime}$ defined by Eq. $[20$. Such conjugate pairs are similar to those considered in [34].

The definition of the heat flux could appear to be somewhat arbitrary since it does not change the physical results 44. However, as it leads to different conjugate pairs, this choice determines in some extent the meaning 
of the physical effects that are described by the linear phenomenological relationships. Keeping that in mind, we assume in this work that the heat flux defined by Eq. 20 and its associated entropy source term given by Eq. 22 are suitable (or at least as suitable as any other) for describing the system of interest.

Now, assuming that the physical system is isotropic, the Curie principle applies [44] and we can propose the following relations:

$$
\begin{aligned}
J_{\varphi} & =L_{\varphi \varphi} \frac{\rho \eta}{T} \\
-\mathbf{q}^{\prime} & =L_{\mathbf{q}^{\prime}, \mathbf{q}^{\prime}} \frac{\nabla T}{T^{2}}+\sum_{i=1}^{n} L_{\mathbf{q}^{\prime}, i} \frac{\nabla\left(\tilde{\mu}_{i}+\beta\right)}{T} \\
-\mathbf{j}_{i} & =L_{i, i} \frac{\nabla\left(\tilde{\mu}_{i}+\beta\right)}{T}+L_{i, \mathbf{q}^{\prime}} \frac{\nabla T}{T^{2}} \quad i=1, \ldots, n
\end{aligned}
$$

The coefficient $L_{\mathbf{q}^{\prime}, \mathbf{q}^{\prime}}$ is related to the thermal conductivity $k$ so that with no mass flow, the heat flux $\mathbf{q}^{\prime}$ is given by the well-known Fourier law. This leads to:

$$
L_{\mathbf{q}^{\prime}, \mathbf{q}^{\prime}}=k T^{2}
$$

The coefficients $L_{\varphi, \varphi}$ and $L_{i, i}$ correspond to mobility terms $M_{\varphi} T$ and $\rho M_{i} T$ associated with the phase-field variable $\varphi$ and the $i$ th component, respectively. Onsager's theory [4] states that the cross coefficients $L_{\mathbf{q}^{\prime}, i}$ and $L_{i, \mathbf{q}^{\prime}}$ are equal. The former is related to the Dufour effect i.e. the presence of a heat flux caused by composition gradients. The latter is associated to the Soret effect i.e. the components diffusion under the temperature gradient. They can be expressed as follows:

$$
L_{i, \mathbf{q}^{\prime}}=L_{i, i} Q_{i}
$$

where $Q_{i}$ is the heat of transport coefficient associated with the $i$ th component. Using the « reduced» heat flux given by Eq.25, Eq. 29] may be written as:

$$
L_{i, \mathbf{q}^{\prime}}=L_{i, i} Q_{i}^{\star}
$$

Here-above, $Q_{i}^{\star}$ is the « reduced» heat of transport [48 that includes a kinetic contribution and a thermodynamic contribution [49, 50,51. Similarly to Eq. 25], it can be expressed as:

$$
Q_{i}^{\star}=Q_{i}-\left(\frac{\partial h}{\partial x_{i}}-\boldsymbol{\psi}_{i}\right)
$$

From Eq. 31, it appears that the heat of transport $Q_{i}$ used in Eq.29] corresponds to the kinetic contribution of $Q_{i}^{\star}$ whereas its thermodynamic contribution is rather contained in the thermodynamic force $\left[\nabla\left(\tilde{\mu}_{i}+\beta\right)\right] / T$ that is used in Eqs. $27 b$ and $27 \mathrm{c}$.

As done by Welland \& al. [34 in similar conditions, we assume that temperature gradients are sufficiently large compared to composition gradients so that the Dufour effect may be neglected. The Soret effect is taken into account in our model.

In these conditions, the phenomenological relations Eqs.27 read:

$$
\begin{aligned}
-\mathbf{q}^{\prime} & =k \nabla T \\
-\mathbf{j}_{i} & =\rho M_{i} \nabla\left(\tilde{\mu}_{i}+\beta\right)+\rho M_{i} Q_{i} \frac{\nabla T}{T} \quad i=1, \ldots, n \\
J_{\varphi} & =M_{\varphi} \rho \eta
\end{aligned}
$$

Now, we shall define the Lagrange multiplier $\beta$ to express the fluxes $\mathbf{j}_{i}$. To this end, we use the mass balance constraint Eq. 2] together with Eq. [8b], yielding:

$$
\sum_{i=1}^{n} \nabla \cdot \mathbf{j}_{i}=0
$$

Then, to satisfy Eq. 33], we propose the following sufficient condition:

$$
\sum_{i=1}^{n} \mathbf{j}_{i}=\mathbf{0}
$$


which, combined with Eq. $32 b$, gives:

$$
\nabla \beta=-\frac{1}{\sum_{j=1}^{n} M_{j}}\left[\sum_{i=1}^{n}\left(M_{i} \nabla \tilde{\mu}_{i}+M_{i} Q_{i} \frac{\nabla T}{T}\right)\right]
$$

Now, after some calculations, one can write $\mathbf{j}_{i}$ in the following form:

$$
-\mathbf{j}_{i}=\frac{\rho}{\sum_{i=j}^{n} M_{j}}\left\{\sum_{k=1, k \neq i}^{n} M_{i} M_{k}\left[\nabla\left(\tilde{\mu}_{i}-\tilde{\mu}_{k}\right)+\left(Q_{i}-Q_{k}\right) \frac{\nabla T}{T}\right]\right\} \quad i=1, \ldots, n
$$

Finally, by substituting the phenomenological relations Eq. $[32 a]$, Eq. $[32 c$ and Eq. 36 in Eqs. $(8)$ and by using Eqs. 18, we are able to write the governing equations in the following form:

$$
\begin{aligned}
\frac{\partial \varphi}{\partial t} & =-M_{\varphi} \rho \eta \\
\rho \eta & =\rho \frac{\partial g}{\partial \varphi}-\kappa \nabla^{2} \varphi \\
\frac{\partial x_{i}}{\partial t} & =\nabla \cdot\left\{\frac{1}{\sum_{j=1}^{n} M_{j}} \sum_{k=1, k \neq i}^{n} M_{i} M_{k}\left[\nabla\left(\tilde{\mu}_{i}-\tilde{\mu}_{k}\right)+\left(Q_{i}-Q_{k}\right) \frac{\nabla T}{T}\right]\right\} \quad i=1, \ldots, n \\
\tilde{\mu}_{i} & =\frac{\partial g}{\partial x_{i}}-\frac{\lambda_{i}}{\rho} \nabla^{2} x_{i} \quad i=1, \ldots, n \\
T \frac{\partial \rho s}{\partial t} & =\nabla \cdot(k \nabla T)-\sum_{i=1}^{n} \nabla\left(\tilde{\mu}_{i}+\beta\right) \cdot \mathbf{j}_{i}-\rho \eta \frac{\partial \varphi}{\partial t}
\end{aligned}
$$

Here-above, Eq. $37 a$ and Eq. $37 b$ correspond to the Allen-Cahn equation whereas Eq. $37 c$ and Eq. $37 d]$ can be viewed as Cahn-Hilliard equations where the diffusive driving force is extended to account for the Soret effect. Following [28] and by using the thermodynamic closures given in \$2.1. one can explicitly express the potentials $\eta$ (multiplied by the molar density $\rho$ ) and $\tilde{\mu}_{i}$ as:

$$
\begin{aligned}
& \tilde{\mu}_{i}=\frac{\partial g^{s}}{\partial x_{i}^{s}}-\frac{\lambda_{i}}{\rho} \nabla^{2} x_{i} \\
& \rho \eta=-\rho h^{\prime}(\varphi)\left[g^{l}-g^{s}-\sum_{i=1}^{n} \frac{\partial g^{s}}{\partial x_{i}^{s}}\left(x_{i}{ }^{l}-x_{i} s\right)\right]+W^{\prime}(\varphi)-\kappa \nabla^{2} \varphi
\end{aligned}
$$

We refer the interested reader to Appendix Affor more details about the derivation of Eqs. 38). More particularly, we show in Appendix A that the potentials $\eta$ (multiplied by the molar density $\rho$ ) and $\tilde{\mu}_{i}$ can be written in the following equivalent form:

$$
\begin{aligned}
& \tilde{\mu}_{i}=\frac{\partial g^{l}}{\partial x_{i}^{l}}-\frac{\lambda_{i}}{\rho} \nabla^{2} x_{i} \\
& \rho \eta=-\rho h^{\prime}(\varphi)\left[g^{l}-g^{s}-\sum_{i=1}^{n} \frac{\partial g^{l}}{\partial x_{i}^{l}}\left(x_{i}^{l}-x_{i}^{s}\right)\right]+W^{\prime}(\varphi)-\kappa \nabla^{2} \varphi
\end{aligned}
$$

Regarding Eq. 37e, in practice, it is more appropriate to perform numerical simulations as a function of temperature instead of entropy. The entropy balance is therefore re-written as:

$$
\rho C_{p} \frac{\partial T}{\partial t}=\nabla \cdot(k \nabla T)-\sum_{i=1}^{n}\left[\frac{\partial h}{\partial x_{i}}-\frac{\lambda_{i}}{\rho} \nabla^{2} x_{i}\right] \frac{\partial \rho x_{i}}{\partial t}-\rho\left(\frac{\partial h}{\partial \varphi}-\frac{\kappa}{\rho} \nabla^{2} \varphi\right) \frac{\partial \varphi}{\partial t}
$$


and we refer the reader to Appendix B for more details about the derivation of this heat equation.

Finally, from Eqs. 37] together with Eqs. 38] and Eq. (40), one can write:

$$
\begin{aligned}
\frac{\partial \varphi}{\partial t} & =-M_{\varphi} \rho \eta \\
\rho \eta & =-\rho h^{\prime}(\varphi)\left[g^{l}-g^{s}-\sum_{i=1}^{n} \frac{\partial g^{s}}{\partial x_{i}^{s}}\left(x_{i}^{l}-x_{i}^{s}\right)\right]+W^{\prime}(\varphi)-\kappa \nabla^{2} \varphi \\
\frac{\partial x_{i}}{\partial t} & =\nabla \cdot\left\{\frac{1}{\sum_{j=1}^{n} M_{j}} \sum_{k=1, k \neq i}^{n} M_{i} M_{k}\left[\nabla\left(\tilde{\mu}_{i}-\tilde{\mu}_{k}\right)+\left(Q_{i}-Q_{k}\right) \frac{\nabla T}{T}\right]\right\} \quad i=1, \ldots, n \\
\tilde{\mu}_{i} & =\frac{\partial g^{s}}{\partial x_{i}^{s}}-\frac{\lambda_{i}}{\rho} \nabla^{2} x_{i} \quad i=1, \ldots, n \\
\rho C_{p} \frac{\partial T}{\partial t} & =\nabla \cdot(k \nabla T)-\sum_{i=1}^{n}\left[\frac{\partial h}{\partial x_{i}}-\frac{\lambda_{i}}{\rho} \nabla^{2} x_{i}\right] \frac{\partial \rho x_{i}}{\partial t}-\rho\left(\frac{\partial h}{\partial \varphi}-\frac{\kappa}{\rho} \nabla^{2} \varphi\right) \frac{\partial \varphi}{\partial t}
\end{aligned}
$$

These equations stand for the general expression of our two-phase compositional phase-field model.

\section{Reduction to the uranium-oxygen binary system in a solid/liquid mixture}

Here, the phase-field model derived in $\$ 2$ is applied to a system of interest for nuclear reactors. So, rather than considering a general multi-component system to describe, for instance, a set of volatile fission products, we focus on the uranium-oxygen binary system. In addition, to simplify the problem, we assume that there is no miscibility gap in the solid and liquid phases and consider that the gradient energy coefficients $\lambda_{o}$ and $\lambda_{u}$ are null. Such an assumption is commonly used in solidification applications [19].

However, as shown by the U-O phase diagram of Fig 2 , the binary system can exhibit miscibility gaps (see 《LIQUID-1+LIQUID-2» in the hypostoichiometric region in Fig 2). Simulation of the associated phase segregation effects would require to keep these gradient coefficient terms in the system, as shown by Cardon \& al. [26] in his treatment of liquid phase segregation in the U-O binary system.

For a uranium-oxygen binary system in a solid/liquid mixture, the phase-field model given by Eqs.41] boils down into the following form:

$$
\begin{aligned}
\frac{\partial \varphi}{\partial t} & =-M_{\varphi}\left\{-\rho h^{\prime}(\varphi)\left[g^{l}-g^{s}-\left(x_{o}^{l}-x_{o}^{s}\right)\left(\tilde{\mu}_{o}-\tilde{\mu}_{u}\right)\right]+W^{\prime}(\varphi)-\kappa \nabla^{2} \varphi\right\} \\
\frac{\partial x_{o}}{\partial t} & =\nabla \cdot\left\{\frac{M_{u} M_{o}}{M_{u}+M_{o}}\left[\nabla\left(\tilde{\mu}_{o}-\tilde{\mu}_{u}\right)+\left(Q_{o}-Q_{u}\right) \frac{\nabla T}{T}\right]\right\} \\
\tilde{\mu}_{o}-\tilde{\mu}_{u} & =\frac{\partial g^{s}}{\partial x_{o}^{s}}-\frac{\partial g^{s}}{\partial x_{u}^{s}}, \quad \text { with } x_{u}+x_{o}=1 \\
\rho C_{p} \frac{\partial T}{\partial t} & =\nabla \cdot(k \nabla T)-\left(\frac{\partial h}{\partial x_{o}}-\frac{\partial h}{\partial x_{u}}\right) \frac{\partial \rho x_{o}}{\partial t}-\rho\left(\frac{\partial h}{\partial \varphi}-\frac{\kappa}{\rho} \nabla^{2} \varphi\right) \frac{\partial \varphi}{\partial t}
\end{aligned}
$$

without forgetting the interfacial region defined by:

$$
\begin{aligned}
x_{o} & =h(\varphi) x_{o}^{s}+[1-h(\varphi)] x_{o}^{l} \\
\frac{\partial g^{s}}{\partial x_{o}^{s}}\left(x_{o}^{s}\right)-\frac{\partial g^{s}}{\partial x_{u}^{s}}\left(x_{u}^{s}\right) & =\frac{\partial g^{l}}{\partial x_{o}^{l}}\left(x_{o}^{l}\right)-\frac{\partial g^{l}}{\partial x_{u}^{l}}\left(x_{u}^{l}\right)
\end{aligned}
$$

where the subscripts $\cdot_{u}$ and ${ }_{o}$ refer to uranium and oxygen, respectively. Here-above, it is noteworthy to mention that the relationship Eq. $43 b$ ensures that the chemical potentials are the same in the interfacial region because we focus on a binary system and $x_{o}+x_{u}=1$. 


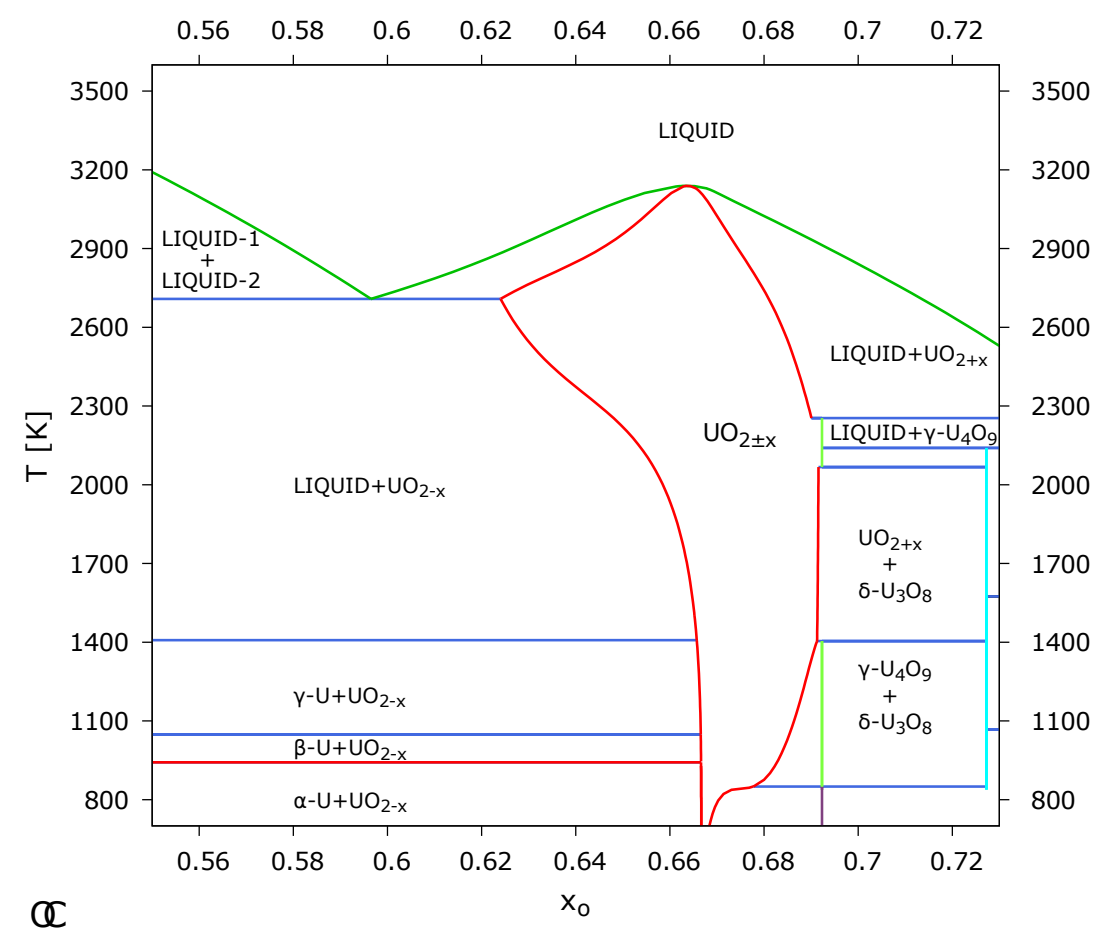

Figure 2: U-O phase diagram calculated by the OpenCalphad thermochemical solver 9 at $5 \mathrm{Mpa}$ (pressure representative of the reactor conditions of interest) with the TAF-ID database [2 39]: visualization in a range of U-O of interest for nuclear reactors.

At this stage, let us take a look at the mobility coefficients whose expressions have not been discussed yet. Following [21] and [52], we express these coefficients in such a way that the two-phase compositional model remains valid in some limiting cases, namely when one of the two phases or one of the two components vanishes. For example, when the solid phase has fully melted (i.e. $\varphi=0$ ), the phase-field model degenerates in the following form:

$$
\begin{aligned}
\frac{\partial x_{o}}{\partial t} & =\nabla \cdot\left[M_{c}^{l}\left(x_{o}, x_{u}\right) \nabla\left(\tilde{\mu}_{o}-\tilde{\mu}_{u}\right)+M_{T}^{l}\left(x_{o}, x_{u}\right) \frac{\nabla T}{T}\right] \\
\tilde{\mu}_{o}-\tilde{\mu}_{u} & =\frac{\partial g^{l}}{\partial x_{o}^{l}}-\frac{\partial g^{l}}{\partial x_{u}^{l}} \\
\rho C_{p} \frac{\partial T}{\partial t} & =\nabla \cdot(k \nabla T)-\left(\frac{\partial h}{\partial x_{o}}-\frac{\partial h}{\partial x_{u}}\right) \frac{\partial \rho x_{o}}{\partial t}
\end{aligned}
$$

where we have related the mobility coefficients $M_{u, u}$ and $M_{o, o}$ to a mobility coefficient $M_{c}^{l}\left(x_{o}, x_{u}\right)$ of the uraniumoxygen binary system in the liquid phase. We have also denoted by $M_{T}^{l}\left(x_{o}, x_{u}\right)$ the product of $M_{c}^{l}\left(x_{o}, x_{u}\right)$ with the difference of the heat of transport coefficients $Q_{o}^{l}-Q_{u}^{l}$ evaluated in the liquid phase. Here-above, Eqs. 44) correspond to the oxygen thermal diffusion problem in the pure liquid phase. This limiting case is obtained whether $M_{\varphi}$ is constant or depends on $\varphi$. So, as an initial approach, we assume $M_{\varphi}$ is constant for numerical reasons, i.e. to ensure the convergence of the numerical method used to solve the discrete form of the Allen-Cahn equation and to guaranty that the movement of the liquid-solid interface is controlled by the compositional diffusion gradient. A similar approach has been followed by Welland [32]. However, in theory, this kinetic parameter should be estimated on the basis of a rigorous dimensional analysis, as proposed by Tiwari [29]. Similarly, when $\varphi=1$, we can link the mobility coefficients to the compositional $M_{c}^{s}\left(x_{o}, x_{u}\right)$ and thermal $M_{T}^{s}\left(x_{o}, x_{u}\right)$ mobility coefficients for the uranium-oxygen binary system in the solid phase. In these conditions, we postulate the following general 
relationships:

$$
\begin{aligned}
& M_{c}\left(\varphi, x_{o}, x_{u}\right):=h(\varphi) \times M_{c}^{s}\left(x_{o}, x_{u}\right)+(1-h(\varphi)) \times M_{c}^{l}\left(x_{o}, x_{u}\right) \\
& M_{T}\left(\varphi, x_{o}, x_{u}\right):=h(\varphi) \times M_{T}^{s}\left(x_{o}, x_{u}\right)+(1-h(\varphi)) \times M_{T}^{l}\left(x_{o}, x_{u}\right)
\end{aligned}
$$

where $h(\varphi)$ is the interpolation function given by Eq.6. In the following, we assume that the generic mobility coefficients in the oxygen thermal diffusion Eqs. $42 b$ are equal to $M_{c}\left(\varphi, x_{o}, x_{u}\right)$ and $M_{T}\left(\varphi, x_{o}, x_{u}\right)$. In Eqs. (45), the coefficients $M_{c}^{s}\left(x_{o}, x_{u}\right), M_{c}^{l}\left(x_{o}, x_{u}\right), M_{T}^{s}\left(x_{o}, x_{u}\right)$ and $M_{T}^{l}\left(x_{o}, x_{u}\right)$ must be defined to prevent unphysical apparition of a component not initially present, such that:

$$
\begin{aligned}
& x_{o}\left(t=t_{0}\right)=0 \quad \Rightarrow \quad \forall t>t_{0}, x_{o}(t)=0 \\
& x_{o}\left(t=t_{0}\right)=1 \quad \Rightarrow \quad \forall t>t_{0}, x_{o}(t)=1
\end{aligned}
$$

To fulfill these constraints, we follow [19] and assume that $M_{c}^{s}\left(x_{o}, x_{u}\right)$ and $M_{c}^{l}\left(x_{o}, x_{u}\right)$ are given in the following form:

$$
\begin{aligned}
& M_{c}^{s}\left(x_{o}, x_{u}\right)=\left(M_{u}^{s} x_{o}+M_{o}^{s} x_{u}\right) \times x_{o} \times x_{u} \\
& M_{c}^{l}\left(x_{o}, x_{u}\right)=\left(M_{u}^{l} x_{o}+M_{o}^{l} x_{u}\right) \times x_{o} \times x_{u}
\end{aligned}
$$

where $M_{u}^{l}, M_{o}^{l}, M_{u}^{s}$ and $M_{o}^{s}$ are the uranium and oxygen mobilities, respectively associated with the liquid and solid phases. They will be detailed in $\$ 5$. At this stage, it is interesting to remark that the governing Eqs. (44) applied to the solid phase can be viewed as an extension of the thermal diffusion equations proposed by Simunovic \& al. [11] where the diffusive driving force only depends on the oxygen chemical potential. Indeed, the diffusive driving force in Eq.(44a) shows that, in our model, the oxygen transport is by oxygen-uranium inter-diffusion and by Soret effect.

Now, we focus on Eq. $42 a$ ) where we must first define the height of the double-well $\omega$ on which $W^{\prime}(\varphi)$ depends and then the gradient energy term $\kappa$. To do so, a standard way consists in conducting an one-dimensional analysis of the phase-field model at the equilibrium state (see for instance [26, 28, 29,32]). Here, it is noteworthy to mention that such an analysis is only valid because the gradient energy coefficients $\lambda_{o}$ and $\lambda_{u}$ are null. Otherwise, the analysis is broken by the inclusion of gradient energy terms (i.e. $\nabla x_{o}$ and $\nabla x_{u}$ ) which produce implicit energy contributions in interfacial energies. As shown in Appendix $\mathrm{C}$ this analysis yields:

$$
\begin{aligned}
& \kappa=\frac{3}{2} \sigma \epsilon \\
& \omega=12 \frac{\sigma}{\epsilon}
\end{aligned}
$$

where $\epsilon$ and $\sigma$ denote the liquid-solid interface thickness (m) and the surface tension $\left(\mathrm{J} \cdot \mathrm{m}^{-2}\right.$ ) acting between these two phases, respectively. They will be defined later for the demonstration problems presented in $\$ 5$

In the heat equation Eq. [44c], the thermal conductivity $k$ and the heat capacity $C_{p}$ depend on temperature but also on the phase composition and the oxygen content. In fuel performance codes, the heat capacity is often calculated according to empirical correlations or given by laws resulting from atomic-scale simulations [53 [54, 55]. It is interesting to keep in mind that $C_{p}$ could be calculated at run-time with a thermochemical solver coupled with a CALPHAD database such as, for instance, OpenCalphad with the TAF-ID.

Finally, one can write the phase-field model in the following form:

$$
\begin{aligned}
\frac{\partial \varphi}{\partial t} & =-M_{\varphi}\left\{-\rho h^{\prime}(\varphi)\left[g^{l}-g^{s}-\left(x_{o}^{l}-x_{o}^{s}\right)\left(\tilde{\mu}_{o}-\tilde{\mu}_{u}\right)\right]\right. \\
& \left.+24 \frac{\sigma}{\epsilon} \varphi(1-\varphi)(1-2 \varphi)-\frac{3}{2} \sigma \epsilon \nabla^{2} \varphi\right\} \\
\frac{\partial x_{o}}{\partial t} & =\nabla \cdot\left[M_{c}\left(\varphi, x_{o}, x_{u}\right) \nabla\left(\tilde{\mu}_{o}-\tilde{\mu}_{u}\right)+M_{T}\left(\varphi, x_{o}, x_{u}\right) \nabla \ln (T)\right] \\
\tilde{\mu}_{o}-\tilde{\mu}_{u} & =\frac{\partial g^{s}}{\partial x_{o}^{s}}-\frac{\partial g^{s}}{\partial x_{u}^{s}}, \quad \text { with } x_{u}+x_{o}=1 \\
\rho C_{p} \frac{\partial T}{\partial t} & =\nabla \cdot(k \nabla T)-\left(\frac{\partial h}{\partial x_{o}}-\frac{\partial h}{\partial x_{u}}\right) \frac{\partial \rho x_{o}}{\partial t}-\left(\rho \frac{\partial h}{\partial \varphi}-\frac{3}{2} \sigma \epsilon \nabla^{2} \varphi\right) \frac{\partial \varphi}{\partial t}
\end{aligned}
$$


where, for numerical convenience, we have reformulated the Soret term in Eq. $49 b$ as a function of the gradient of the logarithm of the temperature (i.e. $1 / T \nabla T=\nabla \ln (T)$ ).

At this stage, it is interesting to discuss the link between the phase-field model and a CALPHAD database. As mentioned in \$1, the goal is to use the thermodynamic data from the TAF-ID [2, 39]. This database provides a complex description of the solid and liquid phases based on sub-lattices models that does not allow to make a direct link to the homogeneous Gibbs energy function of the phase-field model. Indeed, to illustrate the complexity, the variables of the Gibbs energy functions in the sub-lattices models provided by the TAF-ID are the mole fractions of constituents in each sub-lattice (e.g. ion fractions in the liquid ionic model) whereas ours are expressed in mole fraction of the $\mathrm{U}$ and $\mathrm{O}$ elements. So, among the different approaches available in the open literature to interface a CALPHAD thermodynamic description with a phase-field model, our coupling method relies on thermodynamic equilibrium calculations performed with the OpenCalphad thermochemical solver [9]. More particularly, all CALPHAD contributions in Eqs. 49, namely $g^{l}, g^{s}, \tilde{\mu}_{u}\left(=\frac{\partial g^{s}}{\partial x_{u}^{s}}\right), \tilde{\mu}_{o}\left(=\frac{\partial g^{s}}{\partial x_{o}^{s}}\right), x_{o}^{l}, x_{u}^{l}, x_{o}^{s}, x_{u}^{s}, \frac{\partial h}{\partial x_{o}}$ and $\frac{\partial h}{\partial x_{u}}$ will be given by OpenCalphad $\left.\right|^{2}$ 《local» equilibrium calculations performed at each time step and at each node of the mesh. The coupling is detailed in $\$ 4$

\section{Phase-field solver}

The phase-field model given by Eqs. 49, has been implemented in a C++ program. It can be used as a standalone program but also as a dedicated component in the PLEIADES/Fuel Performance Codes to simulate incipient fuel melting and oxygen transport.

This phase-field solver is directly coupled with the OpenCalphad thermochemical solver recently integrated in the PLEIADES platform [13] which relies on the TAF-ID database [2, 39] for equilibrium calculations. The use of a thermochemical solver forces us to perform a partitioned scheme at each time step $(c f$. $\$ 4.1)$. As a first straightforward implementation to well analyze and parameterize the phase-field model, we consider 1D problems, discretized by a Finite-Difference scheme $(c f . \$ 4.2$.

In the following, the numerical treatment of the heat equation Eq. $49 d$ is not discussed. Without loss of generality, the focus is on Eq. $49 a)$, Eq. $49 b$ and Eq. $(49 c)$ assuming the temperature $T$ be known.

\subsection{Partitioned scheme solved at each time step}

As previously explained, the use of a thermochemical solver with a CALPHAD database leads us to develop a partitioned scheme, here solved at each time step by a fixed-point algorithm. Fig 3 presents the solution procedure performed at each iteration of the fixed-point algorithm. It consists in three main stages, starting with the local equilibrium calculations (see «Stage $1 »$ in Fig 3. These calculations are performed with the OpenCalphad thermochemical solver recently integrated in the PLEIADES platform [13. Here, the scope of the numerical strategies proposed in [13] have been expanded for the needs of the current application. Thus, equilibrium calculations are either done in a pure phase (liquid or solid) or in the interfacial region depending on the value of the phaseindicator $\varphi$. As done in [13] to deal with the trace fission products (\$2.4.4 in reference [13]), a preliminary step is added to classify the nodes of the mesh in three groups depending on whether they belong to the solid phase (i.e. $\varphi=1$ ), to the liquid phase (i.e. $\varphi=0$ ) or to the interfacial region (i.e. $\varphi \in] 0,1[$ ). The spatial strategy discussed in $\$ 2.4 .2$ of reference [13] to initialize the thermochemical calculations is then used. There is no technical difficulty in performing equilibrium calculations in the pure phases for the U-O binary system. However, regarding the nodes of the mesh located at the interface, it is a little more tricky since we have to find the liquid and solid compositions that are consistent with the average composition of the mixture and that ensure the equality of the chemical potentials. In other words, we have to solve a non linear problem that can be written as follows. Let $x_{o}$ and $\varphi$ being known, we have to find $x_{o}^{l}$ such that:

$$
F\left(x_{o}^{l}\right)=0
$$

\footnotetext{
${ }^{2}$ For more details about the links between these thermodynamics quantities and the OpenCalphad thermochemical solver, the interested reader may refer to the Eq.22 in reference [8] which defines the molar Gibbs energy. Their values are computed at run-time from the outputs of the OpenCalphad thermochemical solver managed by an application software interface (see $\$ 2.2 .3$ in reference 13 ).
} 


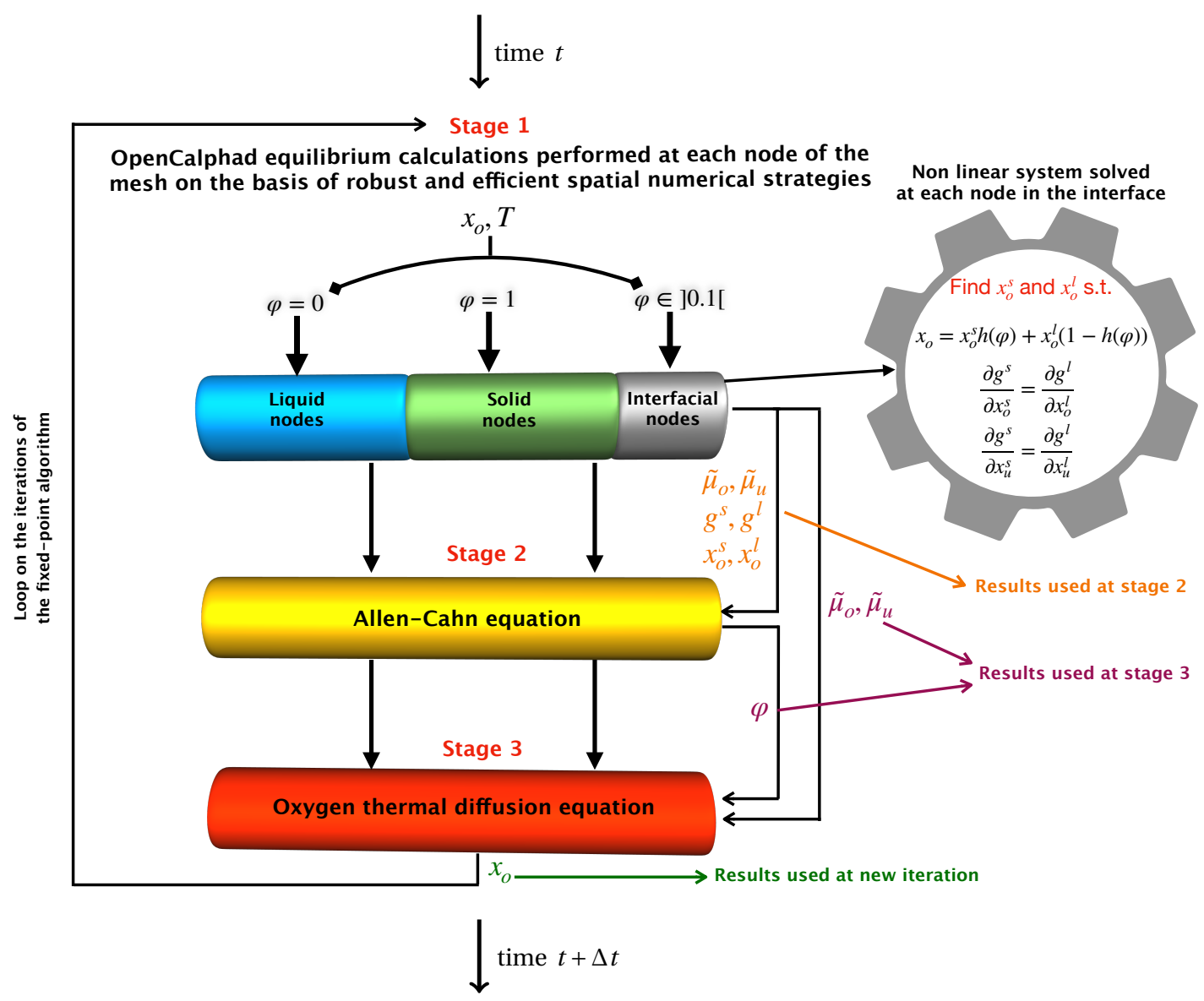

Figure 3: Partitioned scheme solved by a fixed-point algorithm at each time step $\Delta t$.

where $F$ is an univariate function defined by:

$$
F\left(x_{o}^{l}\right)=\left(\frac{\partial g^{s}}{\partial x_{o}^{s}\left(x_{o}^{l}\right)}-\frac{\partial g^{s}}{\partial x_{u}^{s}\left(x_{o}^{l}\right)}\right)-\left(\frac{\partial g^{l}}{\partial x_{o}^{l}}-\frac{\partial g^{l}}{\partial x_{u}^{l}\left(x_{o}^{l}\right)}\right)
$$

Here-above, the chemical potentials are consecutively calculated by the OpenCalphad thermochemical solver in the liquid phase with $x_{o}^{l}$ and in the solid phase with $x_{o}^{s}=\left[x_{o}-(1-h(\varphi)) x_{o}^{l}\right] / h(\varphi)$ whereas the root of Eq. 50) is found with TOMS Algorithm 748 [56]. The algorithm used to perform equilibrium calculations during the first stage is schematically described in Fig 4. As mentioned in this figure, the outputs of interest are the chemical potentials, the Gibbs energies and the oxygen molar fractions in pure phases. They are used to calculate the CALPHAD source term in the Allen-Cahn equation Eq. $49 a$, which is solved during the second stage of the partitioned scheme. The thermodynamic outputs of the first stage are also used with the updated phase-indicator $\varphi$ resulting from the second to estimate the diffusive driving force of the oxygen thermal diffusion equation Eq. $49 b$ (with Eq. (49c). The latter equation is solved during the third stage of the partitioned scheme. At the end of this third stage, the phase-field variable and the oxygen molar fraction are updated and their values can be used to start a new iteration of the fixed-point algorithm. When the convergence of the fixed-point iterations is reached, the phase-field problem can be solved at the next time step using the same procedure. 

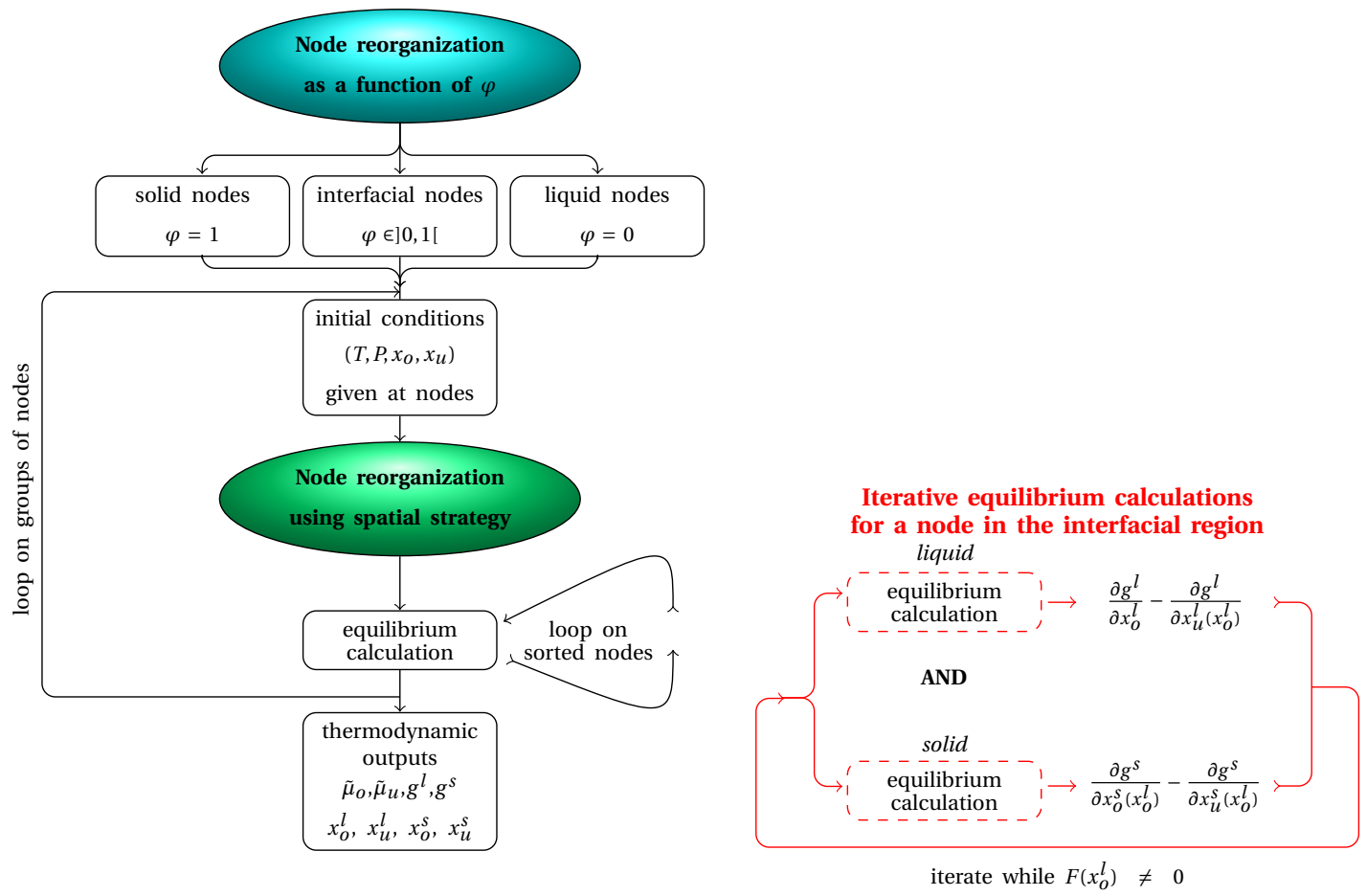

Figure 4: Schematic representation of the proposed algorithm used to perform equilibrium calculations during the first stage of the partitioned scheme

\subsection{Finite-Difference solution of the two-phase compositional problem}

As explained in $\$ 4.1$, after doing all the equilibrium calculations, the two-phase problem and the oxygen thermal diffusion problem are solved sequentially. Even if the time discretization scheme is implicit within each equation, the problem splitting (partitioned resolution) involves a semi-implicit resolution of the global problem variables. Therefore, this may lead to a time step restriction depending for instance on the magnitude of the mobility coefficients, the temperature, the chemical potentials and the space size. Regarding the space discretization, we focus here on one-dimensional problem solved by centered Finite Differences. The interested reader may refer to Appendix D for more details about the derivation of this numerical scheme.

\section{Demonstration problems}

In this section, we present two demonstration problems based on applications to nuclear fuel already studied in open literature. The range of $\mathrm{U}-\mathrm{O}$ of interest for these two demonstration problems is shown in the phase diagram depicted in Fig[5] Following Simunovic \& al. [11], we begin by simulating the oxygen transport in $\mathrm{UO}_{2.005}$ under an imposed thermal gradient with a maximum temperature lower than the liquidus. This numerical experiment aims at verifying the thermodynamic consistency of the phase-field model when one of the two phases is initially absent and at assessing the impact of the formulation of the diffusive driving forces on the results. Then, based on the work proposed by Welland \& al. [33], we check the robustness and the capability of our phase-field model to simulate incipient melting and oxygen transport in oxidized fuel under an increasing thermal gradient. $O / U$ equal to 2.01 and 2.05 


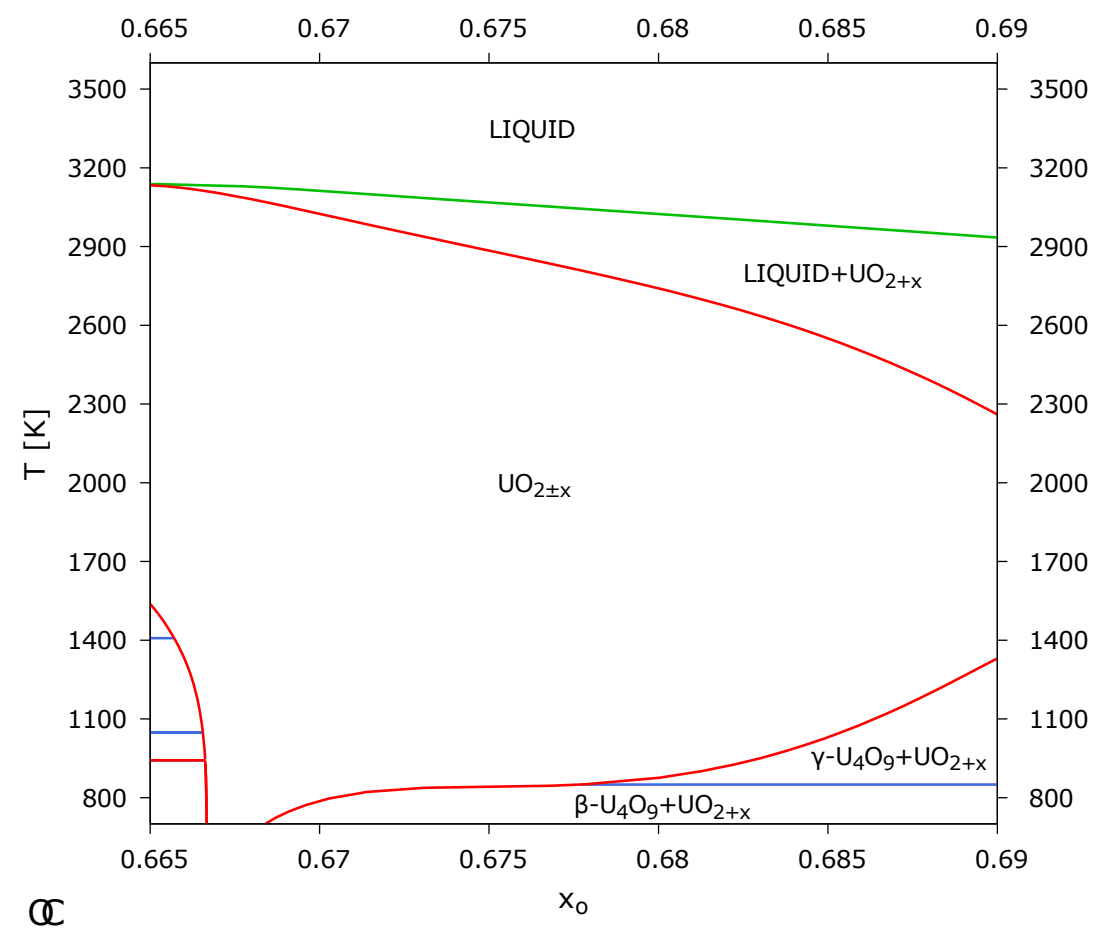

Figure 5: U-O phase diagram calculated by the OpenCalphad thermochemical solver [9] at 5Mpa (pressure representative of the reactor conditions of interest and used for the demonstration problems) with the TAF-ID database [2] 39]: focus on the hyperstoichiometric region.

Before presenting the results, let us define the kinetics parameters of the models and the computational domain used for these two tests.

\subsection{Kinetics parameters}

As previously explained, the CALPHAD terms in our phase-field model result from OpenCalphad equilibrium calculations done with the thermodynamic data of the TAF-ID. More particularly, the liquid phase is thermodynamically described by a single ionic two-sublattice model [2, 42] while the solid phase of mixed oxides with a fluorite structure is described by a three-sublattice model [2]. In case of a uranium-oxygen binary system, it reduces to the following description:

$$
\left(\mathrm{U}^{3+}, \mathrm{U}^{4+}, \mathrm{U}^{5+}\right)_{1}\left(\mathrm{O}^{2-}, \mathrm{Va}\right)_{2}\left(\mathrm{O}^{2-}, \mathrm{Va}\right)_{1}
$$

where the first sublattice corresponds to the metallic cations, the second to the normal site for oxygen anions while the third sublattice represents the interstitial oxygen anions in the fluorite phase [2, 39]. Moore \& al. [35] have related the concentration of defects in $\mathrm{UO}_{2 \pm x}$ (i.e. the site fractions of each component in each sublattice) to the stoichiometric deviation and developed a model of oxygen and uranium diffusion in non-stoichiometric uranium dioxide. For more details about the diffusion mechanisms associated to each component, we refer the interested reader to reference [35. According to their model, the uranium mobility $M_{u}^{s}$ in Eq. $47 a$ ] can be expressed as:

$$
M_{u}^{s}=y_{U^{3+}} M_{U^{3+}} \exp \left(-\frac{Q_{U^{3+}}}{R T}\right)+y_{U^{4+}} M_{U^{4+}} \exp \left(-\frac{Q_{U^{4+}}}{R T}\right)+y_{U^{5+}} M_{U^{5+}} \exp \left(\frac{-Q_{U^{5+}}+y_{U^{4+}} y_{U^{5+}} I_{U^{5+}}}{R T}\right)
$$

where $R$ is the gas constant $\left(\mathrm{J} \cdot \mathrm{mol}^{-1} \cdot \mathrm{K}^{-1}\right)$ and $T$ the temperature. In Eq. (53), the quantities $y$ are the site fractions of cationic species (calculated by the OpenCalphad thermochemical solver) while the parameters $M, Q$ and 


\begin{tabular}{|c|c|c|c|}
\cline { 2 - 4 } \multicolumn{1}{c|}{} & $\mathrm{U}^{3+}$ & $\mathrm{U}^{4+}$ & $\mathrm{U}^{5+}$ \\
\hline $\mathrm{M}\left[\mathrm{mol} \cdot \mathrm{m}^{2} \cdot \mathrm{J}^{-1} . \mathrm{s}^{-1}\right]$ & $1.55 \times 10^{-8}$ & $5.5 \times 10^{-9}$ & $1.35 \times 10^{-9}$ \\
\hline $\mathrm{Q}\left[\mathrm{kJ} \cdot \mathrm{mol}^{-1}\right]$ & 540 & 580 & 440 \\
\hline $\mathrm{I}\left[\mathrm{kJ} \cdot \mathrm{mol}^{-1}\right]$ & - & - & 345 \\
\hline
\end{tabular}

Table 1: Pre-exponential factors and activation energies associated with the uranium mobility in the solid phase 35

$I$ denote pre-exponential factors, activation energies and a non-temperature dependent mixing term, respectively. Their values are given in Table 1 The diffusion model proposed in [35] describes the oxygen mobility $M_{o}^{s}$ in Eq. $47 a$ in the following form:

$$
\begin{aligned}
M_{o}^{s} & =y_{V a}\left(1-y_{V a}\right) M_{V a} \exp \left(\frac{-Q_{V a}+y_{V a}\left(1-y_{V a}\right)\left(A_{V a}-B_{V a} T\right)}{R T}\right) \\
& +y_{I_{o}}\left(1-y_{I_{o}}\right) M_{I_{o}} \exp \left(\frac{-Q_{I_{o}}+y_{I_{o}}\left(1-y_{I_{o}}\right) A_{I_{o}}}{R T}\right)
\end{aligned}
$$

where the subscripts $\cdot \mathrm{Va}$ and $\cdot_{I_{o}}$ correspond to quantities associated with the oxygen vacancy in the second sublattice and the interstitial oxygen anions in the third sublattice, respectively. The pre-exponential factors $M$, the activation energies $Q$ and the mixing terms $A$ and $B$ are given in Table2. Regarding the liquid phase, the uranium

\begin{tabular}{|c|c|c|}
\cline { 2 - 3 } \multicolumn{1}{c|}{} & $\mathrm{Va}$ & $I_{o}$ \\
\hline $\mathrm{M}\left[\mathrm{mol} \cdot \mathrm{m}^{2} \cdot \mathrm{J}^{-1} \cdot \mathrm{s}^{-1}\right]$ & $4.38 \times 10^{-12}$ & $2.76 \times 10^{-10}$ \\
\hline $\mathrm{Q}\left[\mathrm{kJ} \cdot \mathrm{mol}^{-1}\right]$ & 53.498 & 95.411 \\
\hline $\mathrm{A}\left[\mathrm{kJ} \cdot \mathrm{mol}^{-1}\right]$ & 2.234 & -90.548 \\
\hline $\mathrm{B}\left[\mathrm{kJ} .(\mathrm{mol} \cdot \mathrm{K})^{-1}\right]$ & 0.01991 & - \\
\hline
\end{tabular}

Table 2: Pre-exponential factors and activation energies associated with the oxygen mobility in the solid phase [35] (here, $M_{I_{O}}$ has to be multiplied by 100 in order to be consistent with the Fig.6 shown in 35])

and oxygen mobilities $M_{u}^{l}$ and $M_{o}^{l}$ should be defined similarly to $M_{u}^{s}$ and $M_{o}^{s}$. However, to our knowledge, there is no diffusion model for the ionic two-sublattice model used to describe the liquid phase. Consequently, for these demonstration problems, we consider that at each time step, $M_{u}^{l}$ and $M_{o}^{l}$ are equal to the maximum values of $M_{u}^{s}$ and $M_{o}^{s}$ calculated at the hottest point in the solid phase.

As far as the oxygen heat of transport coefficient is concerned, we use as first approximation the correlation recently proposed by Konarski \& al. [5] for the solid phase. It reads:

$$
Q_{o}^{s}=-2.04 \times 10^{-4} \times \exp \left(5.35\left[4+\frac{2\left(\frac{x_{o}}{x_{u}}-2\right)}{0.0508}\right]\right) \text { in } \mathrm{J} . \mathrm{mol}^{-1}
$$

To the best of our knowledge, no reference data is available in the open literature for the uranium heat of transport. Consequently, $Q_{u}^{s}$ is null for these demonstration problems. Given the lack of data, Welland \& al. [34] ignored the Soret effect in the liquid phase in their numerical experiments. Here, similarly to [34], we consider that the difference of the heat of transport coefficients $Q_{o}^{l}-Q_{u}^{l}$ is zero in the pure liquid phase.

At this stage, the interfacial energy $\sigma$, the interface thickness $\epsilon$ and the mobility coefficient $M_{\varphi}$ needs to be defined. We consider $\sigma=6.10^{-2} \mathrm{~J} \cdot \mathrm{m}^{-2}$ according to [32] and we choose $\epsilon=3.10^{-4} \mathrm{~m}$ and $M_{\varphi}=10^{-4}$. As mentioned in $\$ 3$ this value is chosen for numerical convenience but it should be estimated on the basis of a rigorous dimensional analysis [29].

\subsection{Computational domain and solution algorithm}

Fig 6 shows the computational domain $\Omega$ with its boundaries $\Gamma_{0}$ (center of the fuel pellet) and $\Gamma_{N}$ (periphery of the fuel pellet) that is used for both demonstration problems. It corresponds to a half fuel pellet with a radius 
$R=4.65 \mathrm{~mm}$. Regarding the discrete problem, $\Omega$ is described by an uniform grid composed of 41 points (i.e grid similar to those that are commonly used to perform one dimensional simulations with Fuel Performance Codes [57) and then, 5 grid points in the liquid-solid interface, sufficient to have a precise resolution without increasing the computational cost too much.

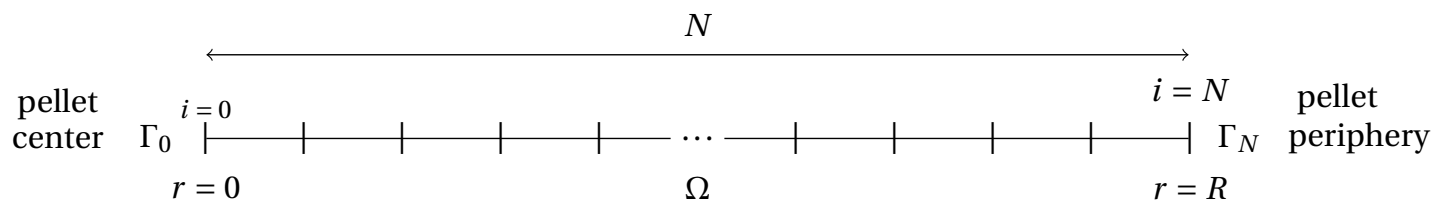

Figure 6: Schematic representation of the computational domain $\Omega=[0, R]$ with its boundaries $\Gamma_{0}$ and $\Gamma_{N}$

In this work, we consider it is sufficient to perform only one iteration of the fixed-point algorithm described in $\$ 4.1$ to solve accurately these $1 \mathrm{D}$ demonstration problems. In that case, the partitioned scheme solved at each time step reduces to a one way coupling scheme. The time step used for the first problem is equal to $10^{-1} \mathrm{~s}$. The harsh conditions associated to melting in the second problem require smaller time steps. Therefore, we have used $\Delta t=2 \times 10^{-2}$ s and $\Delta t=2 \times 10^{-3}$ s for the lowest and highest fuel oxidation levels, respectively.

\subsection{Simulation of oxygen transport in $U_{2.005}$ under an imposed radial temperature gradient below the liquidus}

In this section, we simulate the oxygen transport in hyperstoichiometric fuel under an imposed temperature gradient below the liquidus. In this case, $\varphi=1$ and the phase-field model reduces to the oxygen thermal diffusion equation in the solid phase. This demonstration problem aims at checking the consistency of the model but also assessing the impact of the Soret effect in the solid phase on the oxygen redistribution along the fuel pellet radius. To do so, we have performed two simulations without and with accounting for the temperature gradient in the diffusive driving force. In the following, these simulations are referred to as «diffusion case» and «thermal diffusion case », respectively.

Regarding temperature, we consider a parabolic profile similar to the one proposed in [58]. It reads:

$$
T(r)=T(R)+P_{0} \times\left(\frac{R^{2}-r^{2}}{4 \pi R^{2} k}\right)
$$

where $k=2 \mathrm{~W} \cdot \mathrm{m}^{-1} \cdot \mathrm{K}^{-1}$ is the thermal conductivity and $T(R)=835 \mathrm{~K}$ the temperature imposed on $\Gamma_{N}(r=R)$ and $P_{0}$ the linear heat rate calculated to have $1873 \mathrm{~K}$ imposed on $\Gamma_{0}$ (i.e. $P_{0} \simeq 26 \mathrm{~kW} \cdot \mathrm{m}^{-1}$ ). The radial distribution of temperature obtained with Eq. [56] is depicted in Fig]7. It is representative of temperature profiles induced by linear heat rates encountered during power ramps.

Figs 8 show the initial state of the simulations in terms of oxygen-to-uranium ratio $O / U$, chemical potential difference $\tilde{\mu}_{o}-\tilde{\mu}_{u}$ and oxygen chemical potential $\tilde{\mu}_{o}$. In Fig $8 \mathrm{a}$ we can see that the $\mathrm{O} / \mathrm{U}$ ratio of the fuel is uniform along the pellet radius and equal to 2.005. The graphs of Fig $8 \mathrm{~b}$ and Fig $8 \mathrm{c}$ show that the chemical potentials $\tilde{\mu}_{o}-\tilde{\mu}_{u}$ and $\tilde{\mu}_{o}$ decrease from the periphery to the center of the pellet while temperature increases. The radial profile of the oxygen chemical potential shown in Fig $8 \mathrm{c}$ is in good agreement with the one obtained by Simunovic \& al. (see Fig.5 in the reference [11]).

Figs 9 present the simulation results at steady state. In the graphs, the blue curves refer to the diffusion case whereas the green curves refer to the thermal diffusion case. The radial profiles of the $O / U$ ratio clearly show that the oxygen moved from the periphery (coldest region) to the center of the pellet (hottest region). Moreover, as suggested in [11], we observe that oxygen transport is hardly affected by the Soret effect in the conditions considered for this demonstration problem. This result can be viewed as a direct consequence of the definition of the heat flux in Eq. 20 because the compositional part of the diffusive driving forces is a function of the gradient of the chemical potentials which depend on the temperature implicitly. As discussed in $\$ 2.2$ the thermodynamic contribution of the heat of transport is in some ways included in the gradient of the chemical potentials and only 


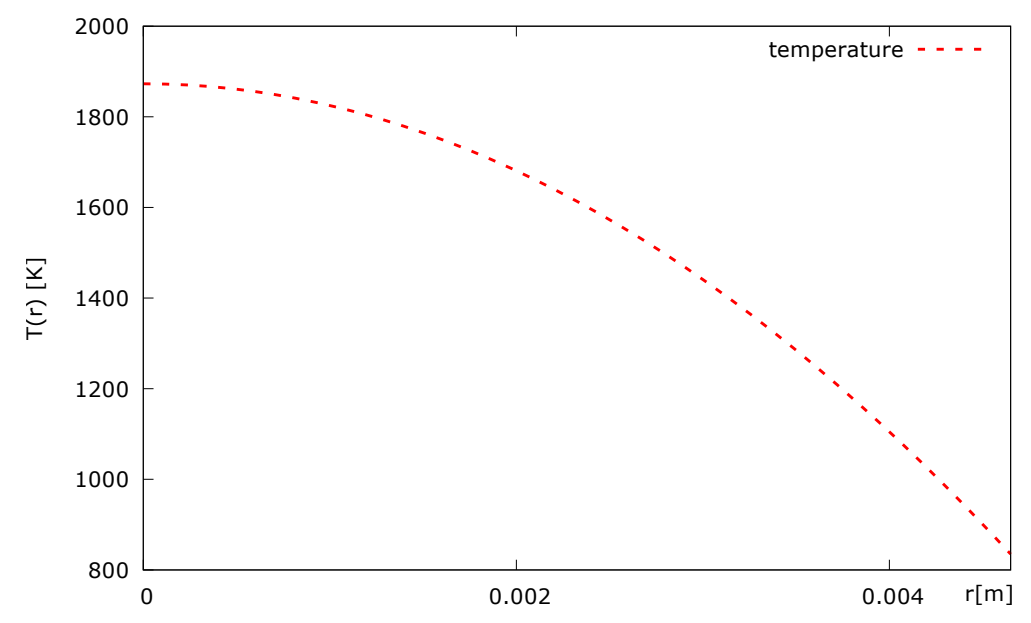

Figure 7: Parabolic temperature profile prescribed along the fuel pellet radius

the kinetic part is associated to the thermal diffusion contribution, here called Soret effect. However, this trend observed under an imposed temperature gradient cannot be generalized to harsher conditions, with higher degree of oxidation and temperature gradients.

Now, let us focus on the radial distribution of the chemical potentials presented in the two last graphs of Fig 9 As expected from Eq. 49], at the steady state, the difference between the oxygen and the uranium chemical potentials becomes uniform for the diffusion case and proportional to the logarithm of the temperature for the thermal diffusion case.

Contrary to the results obtained by Simunovic \& al. (Fig.5 in reference [11]), the oxygen potential remains non uniform along the fuel pellet radius, even for the pure compositional diffusion case. This difference in results is explained simply by the definition of the compositional part of the oxygen equation which describes oxygenuranium inter-diffusion in our model and only oxygen diffusion in the model proposed by Simunovic \& al. [11]. Our results point out that the contribution of uranium cannot be ignored from the diffusion process in order to be thermodynamically consistent with the equilibrium calculations done for the uranium-oxygen binary system. Consequently, to simulate oxygen diffusion in a multicomponent system [11], it seems more thermodynamically consistent to express the compositional part of the diffusive driving forces as a function of the chemical potentials of all the components involved in the system. Such a formulation is provided by the general form of our phasefield model Eqs. 41). That being said, it should be kept in mind that there is very few experimental measurements of the oxygen chemical potential under these high temperature conditions. The validity of the considered diffusive driving force cannot therefore be assessed for sure. Note furthermore that the proposed formulation of U-O interdiffusion assumes that each $\mathrm{O}$ atom is replaced by an $\mathrm{U}$ atom. At moderate to high temperatures, this assumption leads to an overestimation of $U$ migration in the fuel which is usually not mobile compared to O. As illustrated in the next part, the proposed phase-field formulation aims at describing incipient melting where one might expect a much higher $\mathrm{U}$ mobility more consistent with the proposed formulation of $\mathrm{O}-\mathrm{U}$ inter-diffusion. 


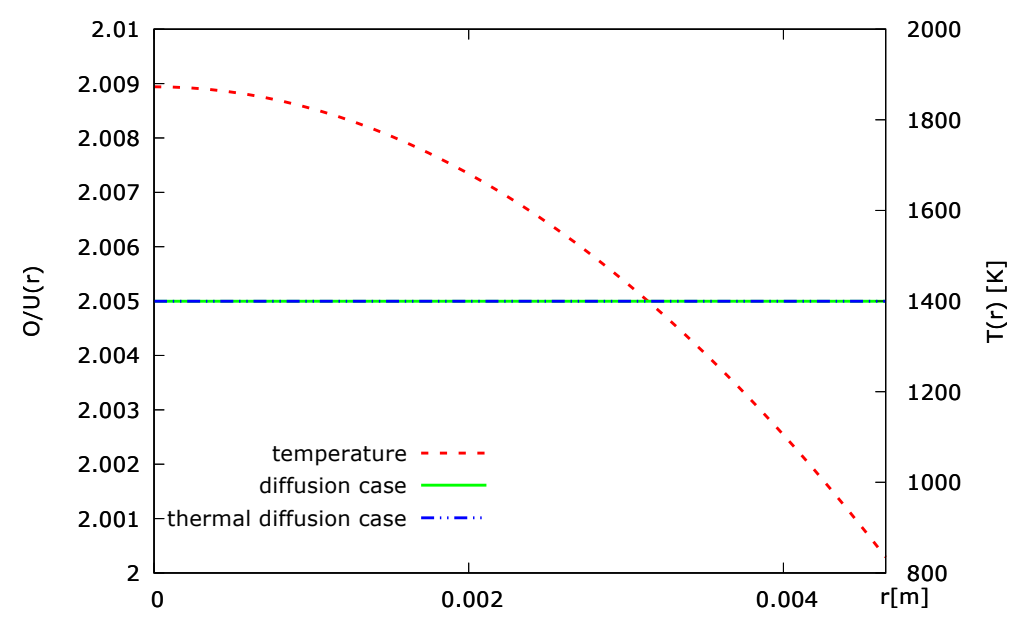

(a) oxygen-to-uranium ratio $O / U \nu s$. pellet radius $r$ at initial state

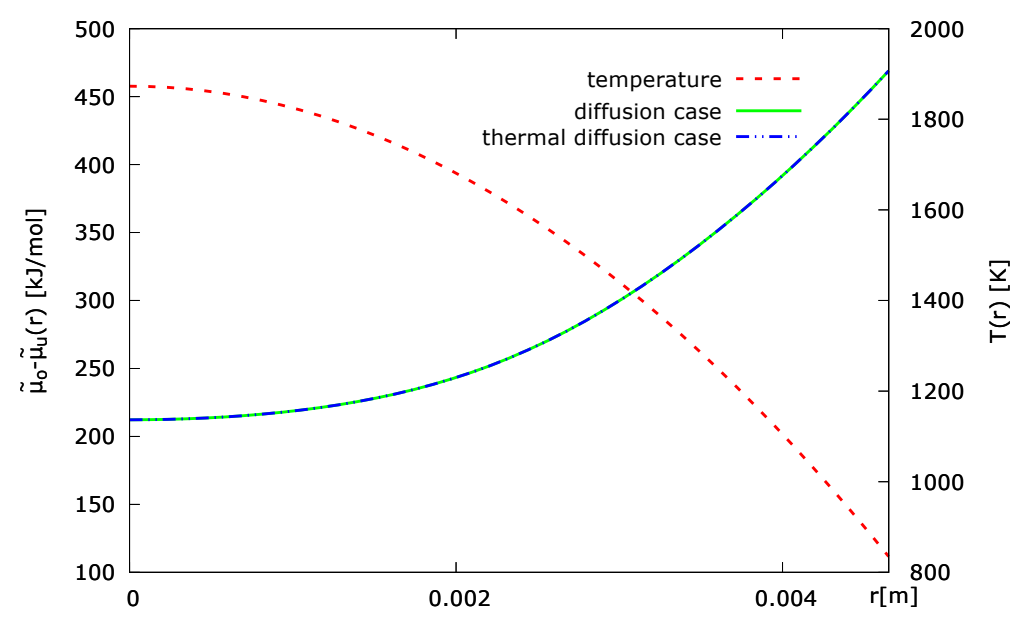

(b) Chemical potentials difference $\tilde{\mu}_{o}-\tilde{\mu}_{u} v s$. pellet radius $r$ at initial state

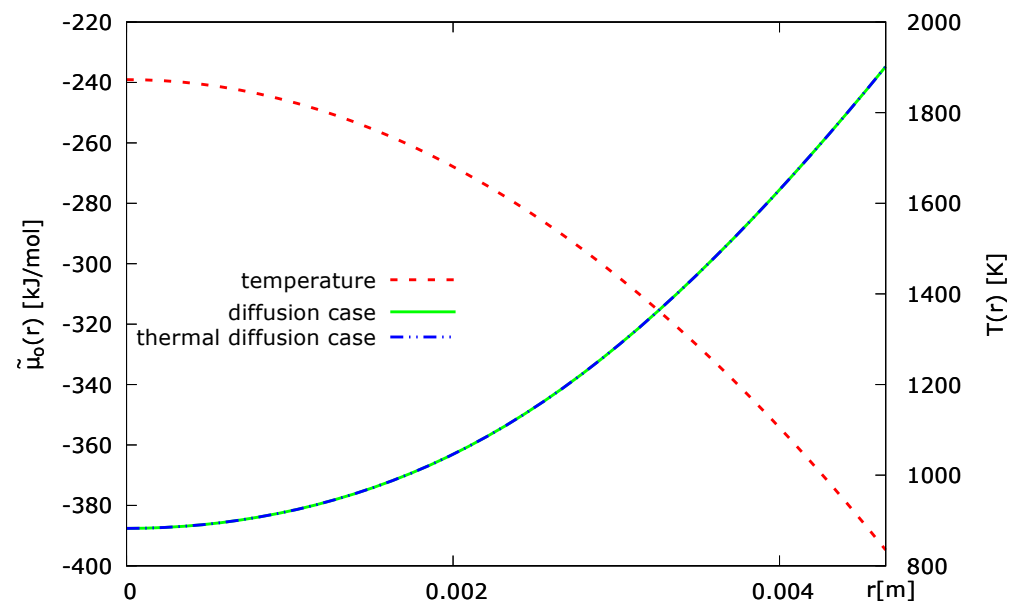

(c) oxygen chemical potential $\tilde{\mu}_{o} \nu s$. pellet radius $r$ at initial state

Figure 8: Radial distribution of $O / U, \tilde{\mu}_{o}-\tilde{\mu}_{u}$ and $\tilde{\mu}_{o}$ along the pellet radius $r$ calculated at the initial state for the simulations done with (diffusion case) and without the Soret effect (thermal diffusion case). The temperature profile is also indicated. 


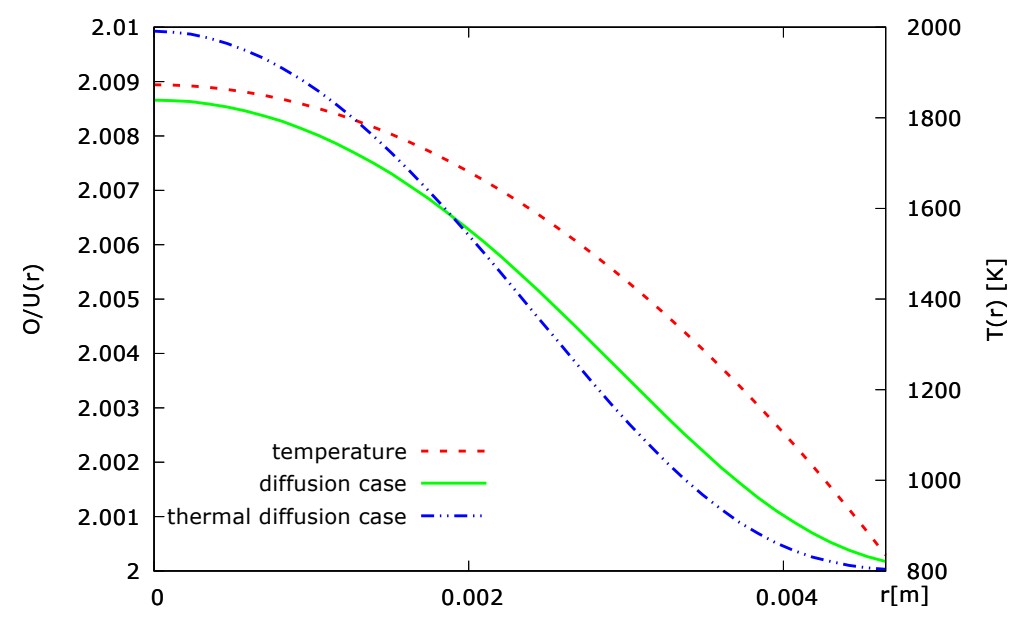

(a) oxygen-to-uranium ratio $O / U \nu$ s. pellet radius $r$ at steady state

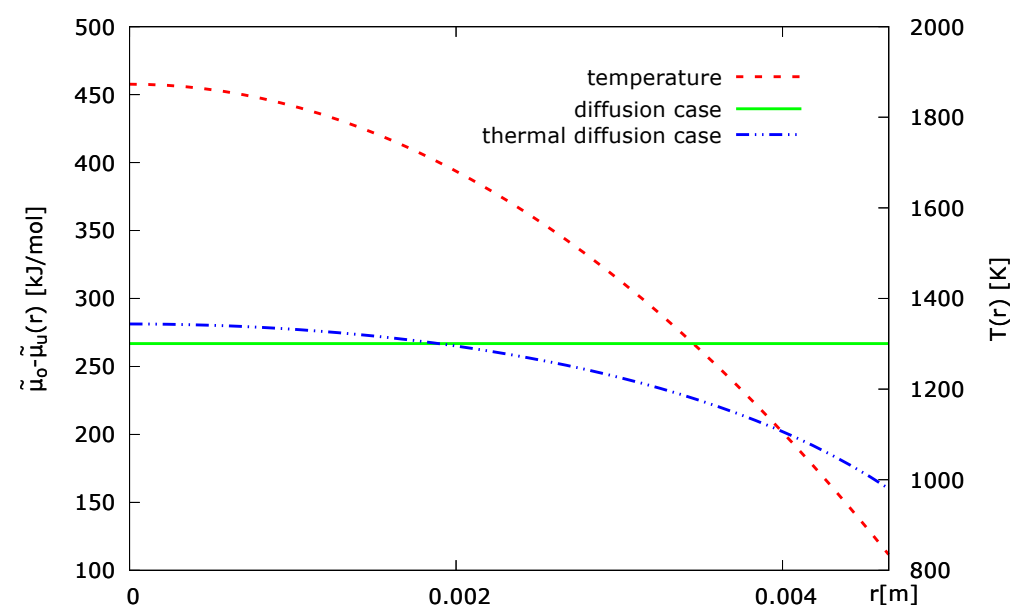

(b) Chemical potentials difference $\tilde{\mu}_{o}-\tilde{\mu}_{u} v s$. pellet radius $r$ at steady state

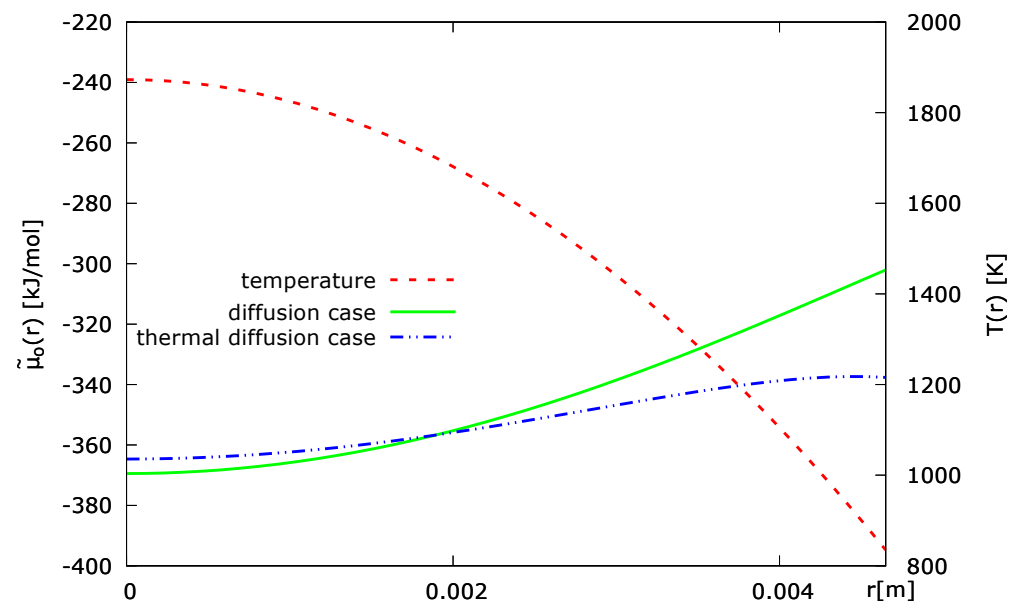

(c) oxygen chemical potential $\tilde{\mu}_{o} v s$. pellet radius $r$ at steady state

Figure 9: Radial distribution of $O / U, \tilde{\mu}_{o}-\tilde{\mu}_{u}$ and $\tilde{\mu}_{o}$ along the pellet radius $r$ calculated at the steady state for the simulations done with (diffusion case) and without the Soret effect (thermal diffusion case). The temperature profile is also indicated. 


\subsection{Simulation of incipient melting and oxygen transport in oxidized fuel under an increasing thermal gradient}

In this section, following [34], we apply our phase-field model to simulate incipient melting together with oxygen transport in oxidized fuel under an increasing thermal gradient. In order to demonstrate the capability of the phase-field model, we perform two simulations with initial oxygen-to-uranium ratios $O / U$ equal to 2.01 and 2.05 .

As done in $\$ 5.3$, we simulate the heat transfer across the oxidized fuel pellet by prescribing a parabolic temperature profile that changes as a function of time $t$ according to:

$$
T(r, t)=T(R)+\left[P_{0}+\min \left(t \times \dot{P}, P_{\text {max }}\right)\right] \times\left(\frac{R^{2}-r^{2}}{4 \pi R^{2} k}\right)
$$

At the initial state, Eq. 57 corresponds exactly to Eq. 56. Here-above, $\dot{P}=900 \mathrm{~W} \cdot \mathrm{m}^{-1} \cdot \mathrm{s}^{-1}$ is the linear power ramp rate allowing us to increase the linear power during more than forty seconds till $P_{\max }=65 \mathrm{~kW} \cdot \mathrm{m}^{-1}$. At the end of this power ramp, the temperature near the center of the pellet reaches $3420 \mathrm{~K}$. The initial and final temperature profiles along the pellet radius are plotted in Fig[10. In this figure, we also present the liquidus in order to show

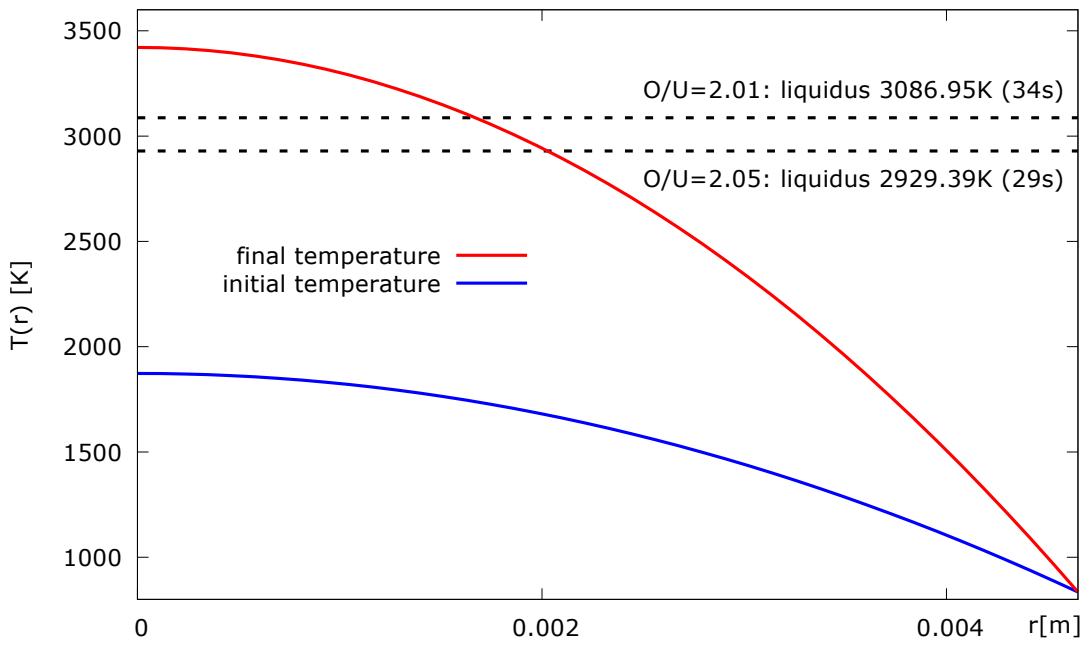

Figure 10: Initial and final temperature profiles prescribed along the fuel pellet radius

the beginning of the melting for both oxidized fuels. This thermodynamic property is automatically calculated at run-time by the OpenCalphad thermochemical solver with the TAF-ID. Phase-field modeling of nucleation (in the present case, the onset of melting) is a complex computational issue out of scope of the present paper. For these simulations, given the high temperatures, we propose to simplify the nucleation problem by assuming that the liquid phase suddenly appears and immediately initiates the fuel melting. From a numerical point of view, this consists in prescribing a Dirichlet-like condition when the liquidus is calculated by OpenCalphad. Here, as expected for one-dimensional simulations performed with the parabolic temperature profile given by Eq. (57), the liquid phase appears first at the hottest grid point of the mesh on $\Gamma_{0}(r=0)$. In practice, we prescribe $\varphi(r=0)=0.1$ when the liquidus is calculated by OpenCalphad. Further parametric studies regarding this arbitrary choice will be considered in a thorough analysis of the model properties. In the case $O / U=2.01$, the fuel melting starts after $34 \mathrm{~s}$ and the liquidus is equal to $3086.95 \mathrm{~K}$. This temperature is lower than the liquidus obtained for stoichiometric fuel, i.e. $3123.50 \mathrm{~K}$, and higher than the liquidus $2929.39 \mathrm{~K}$ calculated for the $O / U=2.05$ fuel that is reached after 29s. These results confirm the trend observed in [34]: the more the fuel is oxidized, the lower the liquidus is and the earlier incipient melting occurs.

The thermodynamic system obtained at the end of the power ramp thus consists of a liquid-solid mixture. At this stage, the highest temperature gradient is maintained during more than 100s in order to study the melting 
front propagation together with the oxygen redistribution process in the two-phase system. Fig 11 and Fig 12 show the results obtained for the fuels with $O / U$ ratios of 2.01 and 2.05, respectively. The radial profiles given in these figures show that the oxygen transport is consistent with the melting front propagation. When the fuel starts to melt, the liquid phase is characterized by a peak of the $O / U$ ratio. Then, the central melted zone extends towards the periphery with a decrease of the $O / U$ ratio in the liquid phase. The fuel oxidation observed in the liquid phase is compensated by a reduction of the oxygen content in the solid phase. Consequently, the local melting temperature raises and the formation of the liquid phase is thus limited. The graphs of Fig 11 and Fig 12 indicate that the melting front reaches a steady state after one hundred seconds. The trends observed in these graphs are in good agreement with those published in [34]. It is however noteworthy that the radial profiles of oxygen in the liquid phase at the end of the calculation and, more particularly, with $O / U=2.01$ slightly differ from those presented in [34]. Indeed, the graphs of Fig 11 suggest that the composition gradient has changed sign in the liquid phase after 50s. A convergence study in time has confirmed these results. This profile can be explained by the small part of the solid phase near the interface where the fuel is hypostoichiometric at 45s and 50s. In that specific case, the oxygen thermal diffusion process is from the center (hottest region) to the periphery (coldest region) [5]. This trend is not seen with $O / U=2.05$ because the fuel remains hyperstoichiometric until the end of the simulation. In that case, the shape of the oxygen distribution obtained in the liquid phase also differs from the one presented in [34] which was uniform (Fig.5 in reference [34). Here, as shown in Fig 12 , the oxygen quantity increases from the liquid-solid interface to the center of the pellet $(r=0)$. This trend seems consistent with the prescribed parabolic temperature profile. To confirm these results, a sensitivity study on the interface thickness and the mobility $M_{\varphi}$ remains to be done.

As a whole, these and the previous results presented in this paper show the capability of our phase-field model to simulate incipient melting and oxygen transport in the fuel with thermodynamics data coming from the TAF-ID. 


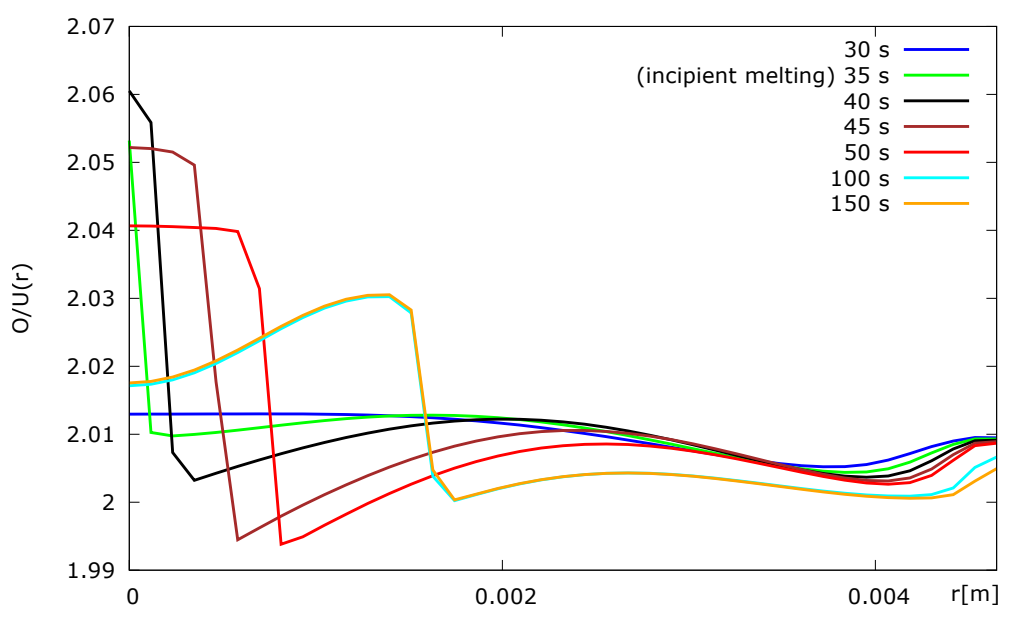

(a) oxygen-to-uranium ratio $O / U \nu$ s. pellet radius $r$

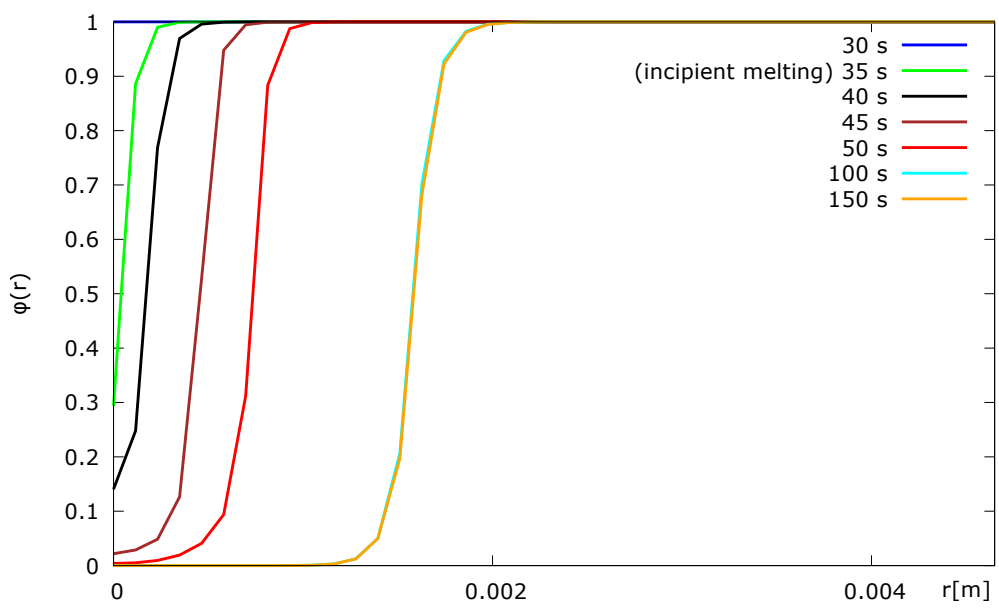

(b) Phase-indicator $\varphi$ vs. pellet radius $r$

Figure 11: Radial distributions of $O / U$ and $\varphi$ along the pellet radius $r$ calculated at several times after detecting incipient melting in the fuel with an initial $O / U=2.01$ 


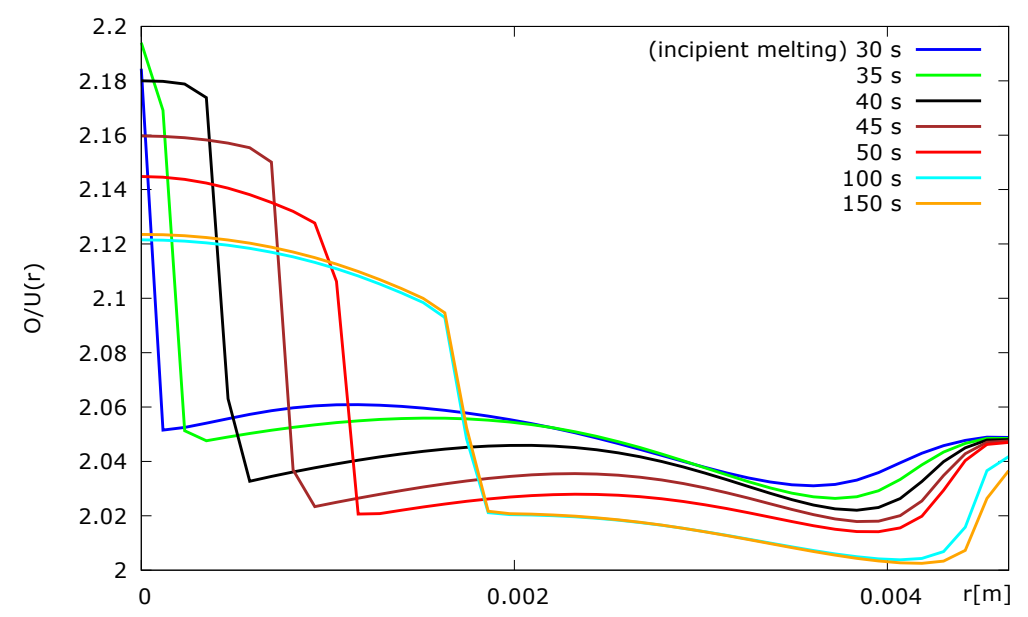

(a) oxygen-to-uranium ratio $O / U \nu s$. pellet radius $r$

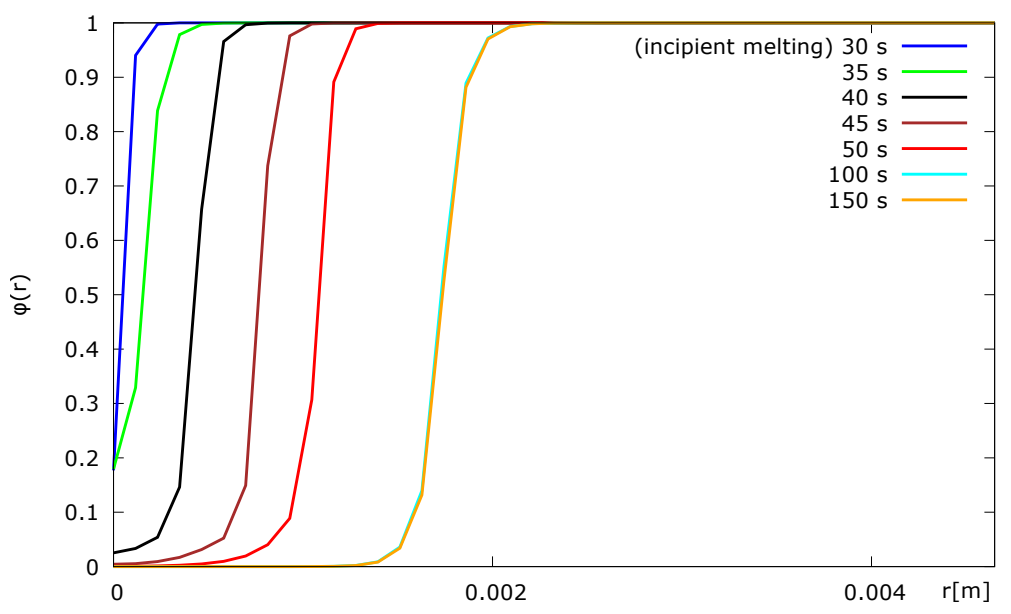

(b) Phase-indicator $\varphi$ vs. pellet radius $r$

Figure 12: Radial distributions of $O / U$ and $\varphi$ along the pellet radius $r$ calculated at several times after detecting incipient melting in the fuel with an initial $O / U=2.05$ 


\section{Conclusion}

The purpose of this work was to improve the modeling included in the PLEIADES platform in order to perform simulations of incipient melting and oxygen transport in the fuel using the thermodynamic properties of the TAFID. To this end, a phase-field model for a two phase compositional (multicomponent) system has been developed and combined with the CALPHAD thermodynamic description of the fuel available in the TAF-ID. The governing equations were first derived within a consistent thermodynamic framework and considering rigorous thermodynamic closures, namely the Gibbs energy of the system and the description of the interfacial region based on the Kim-Kim-Suzuki model. The model was then reduced to a uranium-oxygen binary system in a solid/liquid mixture, of interest in nuclear applications.

In this limiting case, the liquid-solid phase change is described by the Allen-Cahn equation and the oxygen transport by a thermal diffusion equation whose diffusive driving forces include the compositional (oxygenuranium inter-diffusion) and the thermal gradients (Soret effect). The mobility coefficients of the phase-field model were defined to satisfy some consistency properties in the sense that the model had to remain valid when one of the two phases or one of the two components vanishes. The interface with the thermodynamic description of the TAF-ID is performed by coupling the phase-field model with the OpenCalphad thermochemical solver.

Two 1D demonstration problems have been proposed to demonstrate the performance of the numerical scheme. First, oxygen transport in a $\mathrm{UO}_{2.005}$ fuel under an imposed parabolic radial temperature profile below the liquidus temperature has been analyzed. The gradient of the difference between the oxygen and the uranium chemical potentials is used as the main driving force for oxygen diffusion together with the temperature gradient. The radial oxygen redistribution expected for hyperstoichiometric fuel in these thermal conditions is satisfactorily reproduced by the phase-field model. The simulation results clearly show that oxygen redistribution is hardly affected by the Soret effect, as suggested by Simunovic \& al. [11]. However, the contribution of the uranium chemical potential appears non negligible and raises the question of its consideration in oxygen diffusion equations.

The second problem describes incipient melting and oxygen transport in oxidized fuel under an increasing radial thermal gradient. For this demonstration problem, two simulations were performed with initial uniform oxygen-to-uranium ratios equal to 2.01 and 2.05. The simulation results clearly show that the melting front propagation is strongly coupled with oxygen transport in the liquid-solid mixture. As already explained by Welland [34], oxygen redistribution leads to a self-regulating process. Indeed, the oxidation occurring in the liquid phase is compensated by a decrease of the oxygen content in the solid phase, which, in turn, increases the liquidus and stops the fuel melting.

These results demonstrate the consistency of the phase-field model and its capability to simulate incipient melting and oxygen transport with a thermodynamic description of the fuel based on the TAF-ID.

Future work will concern the application of the phase-field model to more complex problems such as power ramps. The numerical scheme could also be adapted in 2D and 3D. The extension of this approach to the uraniumplutonium-oxygen ternary system at hand in Mixed OXide fuels is planned. In that case, the formulation of the phase-field problem may have to be written on the basis of a grand-potential functional [29, 59, 60, 61].

For systems with much larger number of components, the interfacing of an advanced thermodynamic database with a phase-field solver may become a computational issue and thus, the use of an approximate albeit accurate

\section{Acknowledgements}

This work has been carried out within the framework of the PLEIADES platform development funded by CEA, EDF and FRAMATOME. 


\section{Appendix A. Explicit form of the generalized chemical potential}

In this appendix, we present the developments leading to the potentials Eqs. 38]. Following [28], the first step is to calculate the partial derivative of Eqs. (7) with respect to $x_{i}$. It reads:

$$
\begin{aligned}
\frac{\partial g_{0}}{\partial x_{i}} & =h(\varphi) \frac{\partial g^{s}}{\partial x_{i}^{s}} \frac{\partial x_{i}^{s}}{\partial x_{i}}+[1-h(\varphi)] \frac{\partial g^{l}}{\partial x_{i}^{l}} \frac{\partial x_{i}^{l}}{\partial x_{i}} \\
h(\varphi) \frac{\partial x_{i}^{s}}{\partial x_{i}}+[1-h(\varphi)] \frac{\partial x_{i}^{l}}{\partial x_{i}} & =1
\end{aligned}
$$

Then, by substituting the relationships Eq. 7 and Eq. $A .1 b$ in Eq. $A .1 a$, one obtains:

$$
\frac{\partial g_{0}}{\partial x_{i}}=\frac{\partial g^{s}}{\partial x_{i}^{s}}=\frac{\partial g^{l}}{\partial x_{i}^{l}}
$$

which enables to re-write Eq. $37 d$ as:

$$
\tilde{\mu}_{i}=\frac{\partial g^{s}}{\partial x_{i}^{s}}-\frac{\lambda_{i}}{\rho} \nabla^{2} x_{i}
$$

or, by using Eq.A.2, in the following equivalent form:

$$
\tilde{\mu}_{i}=\frac{\partial g^{l}}{\partial x_{i}^{l}}-\frac{\lambda_{i}}{\rho} \nabla^{2} x_{i}
$$

Now, we focus on the potential $\eta$ (multiplied by the molar density $\rho$ ) given by Eq. $37 b$. The derivative of relationships Eq. [5] and Eq. (7) with respect to $\varphi$ gives:

$$
\begin{aligned}
\frac{\partial g_{0}}{\partial \varphi} & =h^{\prime}(\varphi)\left(g^{s}-g^{l}\right)+h(\varphi) \sum_{i=1}^{n} \frac{\partial g^{s}}{\partial x_{i}^{s}} \frac{\partial x_{i}^{s}}{\partial \varphi}+[1-h(\varphi)] \sum_{i=1}^{n} \frac{\partial g^{s}}{\partial x_{i}^{l}} \frac{\partial x_{i}^{l}}{\partial \varphi} \\
h(\varphi) \frac{\partial x_{i}^{s}}{\partial \varphi}+[1-h(\varphi)] \frac{\partial x_{i}^{l}}{\partial \varphi} & =h^{\prime}(\varphi)\left(x_{i}^{l}-x_{i}^{s}\right)
\end{aligned}
$$

Next, by using Eq. $A .5 b$, one can simplify Eq. $A .5 a$ as follows:

$$
\frac{\partial g_{0}}{\partial \varphi}=h^{\prime}(\varphi)\left(g^{s}-g^{l}\right)-\sum_{i=1}^{n} \frac{\partial g^{s}}{\partial x_{i}^{s}} h^{\prime}(\varphi)\left(x_{i}^{s}-x_{i}^{l}\right)
$$

which enables to express the potential $\eta$ (multiplied by the molar density $\rho$ ) as:

$$
\rho \eta=-\rho h^{\prime}(\varphi)\left[g^{l}-g^{s}-\sum_{i=1}^{n} \frac{\partial g^{s}}{\partial x_{i}^{s}}\left(x_{i}^{l}-x_{i}^{s}\right)\right]+W^{\prime}(\varphi)-\kappa \nabla^{2} \varphi
$$

Here again, by using Eq.A.2, Eq. A.7 can be written in the following equivalent form:

$$
\rho \eta=-\rho h^{\prime}(\varphi)\left[g^{l}-g^{s}-\sum_{i=1}^{n} \frac{\partial g^{l}}{\partial x_{i}^{l}}\left(x_{i}^{l}-x_{i}^{s}\right)\right]+W^{\prime}(\varphi)-\kappa \nabla^{2} \varphi
$$

At this stage, as the pure molar Gibbs energies $g^{s}$ and $g^{l}$ are homogeneous functions, it is interesting to note that the CALPHAD contributions in Eq. A.7) and Eq. A.8 can be calculated according to:

$$
\rho\left[g^{l}-g^{s}-\sum_{i=1}^{n} \frac{\partial g^{l}}{\partial x_{i}^{l}}\left(x_{i}^{l}-x_{i}^{s}\right)\right]=\left[g^{l}\left(\rho x_{i}^{l}\right)-g^{s}\left(\rho x_{i}^{s}\right)-\sum_{i=1}^{n} \frac{\partial g^{l}\left(\rho x_{i}^{l}\right)}{\partial \rho x_{i}^{l}}\left(\rho x_{i}^{l}-\rho x_{i}^{s}\right)\right]
$$




\section{Appendix B. Derivation of the heat equation from the entropy balance equation}

In this appendix, we detail the derivation of the heat equation Eq.40 from the entropy balance equation Eq. $37 e$.

To begin, we recall the reader that the molar entropy is defined from the molar Gibbs energy $g$ according to Eq. (11b) and $g$ depends on $p, T,\left\{x_{i}\right\},\left\{\nabla x_{i}\right\}, \varphi$ and $\nabla \varphi$. In these conditions, according to Eq. [11], one can write:

$$
\begin{aligned}
d s & =-\left[\frac{\partial^{2} g}{\partial T \partial p} d p+\frac{\partial^{2} g}{\partial T^{2}} d T+\sum_{i=1}^{n}\left(\frac{\partial^{2} g}{\partial T \partial x_{i}} d x_{i}+\frac{\partial^{2} g}{\partial T \partial \nabla x_{i}} \cdot d \nabla x_{i}\right)+\frac{\partial^{2} g}{\partial T \partial \varphi} d \varphi+\frac{\partial^{2} g}{\partial T \partial \nabla \varphi} \cdot d \nabla \varphi\right] \\
& =-\left[\frac{\partial V_{m}}{\partial T} d p-\frac{\partial s}{\partial T} d T+\sum_{i=1}^{n}\left(\frac{\partial \mu_{i}}{\partial T} d x_{i}+\frac{\partial \boldsymbol{\psi}_{i}}{\partial T} \cdot d \nabla x_{i}\right)+\frac{\partial \theta}{\partial T} d \varphi+\frac{\partial \zeta}{\partial T} \cdot d \nabla \varphi\right]
\end{aligned}
$$

In this work, the molar volume $V_{m}$ is constant and we also assume that the coefficients $\boldsymbol{\psi}_{i}, \zeta$ do not depend on the temperature. In these conditions, by assuming local thermodynamic equilibrium conditions [44, Eq. [B.1] reads:

$$
\frac{\partial s}{\partial t}=-\left[-\frac{C_{p}}{T} \frac{\partial T}{\partial t}+\sum_{i=1}^{n}\left(\frac{\partial \mu_{i}}{\partial T} \frac{\partial x_{i}}{\partial t}\right)+\frac{\partial \theta}{\partial T} \frac{\partial \varphi}{\partial t}\right]
$$

where $C_{p}$ is the heat capacity at constant pressure defined by:

$$
C_{p}=T\left(\frac{\partial s}{\partial T}\right)_{P}
$$

Now, by multiplying Eq. $B .2$ by $\rho T$, we obtain:

$$
T \frac{\partial \rho s}{\partial t}=\rho C_{p} \frac{\partial T}{\partial t}-T \sum_{i=1}^{n}\left(\frac{\partial \mu_{i}}{\partial T} \frac{\partial \rho x_{i}}{\partial t}\right)-\rho T \frac{\partial \theta}{\partial T} \frac{\partial \varphi}{\partial t}
$$

Then, we can introduce the potential term $\tilde{\mu}_{i}+\beta$ in Eq. B.4, yielding:

$$
T \frac{\partial \rho s}{\partial t}=\rho C_{p} \frac{\partial T}{\partial t}-T \sum_{i=1}^{n} \frac{\partial\left(\tilde{\mu}_{i}+\beta\right)}{\partial T} \frac{\partial \rho x_{i}}{\partial t}-\rho T \frac{\partial \theta}{\partial T} \frac{\partial \varphi}{\partial t}
$$

Now, in order to simplify the heat equation, we introduce the molar enthalpy $h$ defined by:

$$
h=g-T\left(\frac{\partial g}{\partial T}\right)_{p, \varphi, \nabla \varphi,\left\{x_{i}\right\},\left\{\nabla x_{i}\right\}}
$$

Then, by using Eqs.(11) together with Eq. 38) and after some calculations, we can write the derivative of Eq. (B.6) with respect to $x_{i}$ in the following form:

$$
\frac{\partial h}{\partial x_{i}}=\left(\tilde{\mu}_{i}+\beta\right)+\frac{\lambda_{i}}{\rho} \nabla^{2} x_{i}-T \frac{\partial\left(\tilde{\mu}_{i}+\beta\right)}{\partial T} \quad i=1, \ldots, n
$$

Similarly, for the phase-field variable $\varphi$, we have:

$$
\frac{\partial h}{\partial \varphi}=\eta+\frac{\kappa}{\rho} \nabla^{2} \varphi-T \frac{\partial \theta}{\partial T}
$$

According to Eq. B.7. and Eq. B.8, Eq. B.5 reads:

$$
T \frac{\partial \rho s}{\partial t}=\rho C_{p} \frac{\partial T}{\partial t}+\sum_{i=1}^{n}\left[\frac{\partial h}{\partial x_{i}}-\left(\tilde{\mu}_{i}+\beta\right)-\frac{\lambda_{i}}{\rho} \nabla^{2} x_{i}\right] \frac{\partial \rho x_{i}}{\partial t}+\rho\left(\frac{\partial h}{\partial \varphi}-\eta-\frac{\kappa}{\rho} \nabla^{2} \varphi\right) \frac{\partial \varphi}{\partial t}
$$


At this stage, we substitute Eq. $37 e$ and Eq. 86 in Eq. $(B .9)$ to obtain the heat equation in the following form:

$$
\rho C_{p} \frac{\partial T}{\partial t}=\nabla \cdot(k \nabla T)-\sum_{i=1}^{n}\left[\frac{\partial h}{\partial x_{i}}-\frac{\lambda_{i}}{\rho} \nabla^{2} x_{i}\right] \frac{\partial \rho x_{i}}{\partial t}-\rho\left(\frac{\partial h}{\partial \varphi}-\frac{\kappa}{\rho} \nabla^{2} \varphi\right) \frac{\partial \varphi}{\partial t}-\nabla \cdot\left[\sum_{i=1}^{n}\left(\tilde{\mu}_{i}+\beta\right) \cdot \mathbf{j}_{i}\right]
$$

In this work, we assume that the latter term on the right-hand-side in Eq. $[B .10]$ is of an order of magnitude lower than the other contributions and thereby, it can be neglected. This estimate can be explained on the basis of a rigorous dimensional analysis but it is out of scope of the present paper.

In these conditions, the heat equation reduces to:

$$
\rho C_{p} \frac{\partial T}{\partial t}=\nabla \cdot(k \nabla T)-\sum_{i=1}^{n}\left[\frac{\partial h}{\partial x_{i}}-\frac{\lambda_{i}}{\rho} \nabla^{2} x_{i}\right] \frac{\partial \rho x_{i}}{\partial t}-\rho\left(\frac{\partial h}{\partial \varphi}-\frac{\kappa}{\rho} \nabla^{2} \varphi\right) \frac{\partial \varphi}{\partial t}
$$

\section{Appendix C. One-dimensional analysis of the phase-field model at the equilibrium state}

In this appendix, our purpose is to define the depth of the double-well potential $\omega$ as a function of the interfacial energy and the interface thickness. To do so, we follow an approach proposed in [28], to cite among others, to conduct an one-dimensional analysis of the Allen-Cahn equation Eq. $42 a$ at the equilibrium state

In these conditions, Eq. $42 a$ reduces to:

$$
\kappa \frac{\partial^{2} \varphi_{e q}(z)}{\partial z^{2}}=-\rho h^{\prime}\left(\varphi_{e q}\right)\left[g^{l}-g^{s}-\sum_{i=1}^{n} \frac{\partial g^{s}}{\partial x_{i}^{s}}\left(x_{i}^{l}-x_{i}^{s}\right)\right]_{e q}+W^{\prime}\left(\varphi_{e q}(z)\right)
$$

where the subscript $\star_{e q}$ denotes quantities evaluated at equilibrium. Now, by multiplying the equation Eq.C.1 by $\frac{\partial \varphi_{e q}}{\partial z}$ and by integrating over $\pm \infty$, one can find:

$$
\left[g^{l}-g^{s}-\sum_{i=1}^{n} \frac{\partial g^{s}}{\partial x_{i}^{s}}\left(x_{i}^{l}-x_{i}^{s}\right)\right]_{e q}=0
$$

which allows us to simplify Eq. C.1] in the following form:

$$
\kappa \frac{\partial^{2} \varphi_{e q}(z)}{\partial z^{2}}=W^{\prime}\left(\varphi_{e q}(z)\right)=2 \omega \varphi_{e q}(z)\left(1-\varphi_{e q}(z)\right)\left(1-2 \varphi_{e q}(z)\right)
$$

Here again, we multiply Eq. C.3] by $\frac{\partial \varphi_{e q}}{\partial z}$ and, afterwards, by integrating over $\pm \infty$, one obtain:

$$
\frac{\partial \varphi_{e q}(z)}{\partial z}=\sqrt{\frac{2 \omega}{\kappa}} \varphi_{e q}(z)\left(1-\varphi_{e q}(z)\right)
$$

where we used $\varphi_{e q}(+\infty)=1$ (solid) and $\varphi_{e q}(-\infty)=0$ (liquid). At this stage, for the sake of simplicity, we consider the following variable change:

$$
v(z)=2 \varphi_{e q}(z)-1
$$

where $v(+\infty)=1$ and $v(-\infty)=0$. Then, the relationship Eq. (C.5) is substituted in Eq. C.4], yieldings:

$$
\frac{\partial v}{1-v(z)^{2}}=\frac{1}{2} \sqrt{\frac{2 \omega}{\kappa}} \partial z
$$

which gives after integrating over $\pm \infty$ :

$$
\operatorname{argtan}(\nu(z))=\frac{1}{2} \sqrt{\frac{2 \omega}{\kappa}} z+K
$$


In Eq. C.7), $K$ is a constant of integration. We can easily show that it is zero by defining the interface location such that $\varphi_{e q}(z=0)=0.5$. Finally, according to Eq.C.5 and Eq. C.7, the phase-field variable at equilibrium is defined by:

$$
\varphi_{e q}(z)=\frac{1}{2}\left[1+\tanh \left(\frac{1}{2} \sqrt{\frac{2 \omega}{\kappa}} z\right)\right]
$$

At this stage, it remains to make links between $\kappa, \omega$, the interfacial thickness $\epsilon$ and the interfacial energy $\sigma$. To do so, we begin by defining $\sigma$ in one-dimension as follows:

$$
\sigma=\int_{-\infty}^{+\infty} \frac{\kappa}{2}\left(\frac{\partial \varphi_{e q}(z)}{\partial z}\right)^{2}+W\left(\varphi_{e q}(z)\right) d z
$$

Then, according to the relationships Eq.C.3 (integrated over $[0,1]$ ) and Eq.C.4, Eq. C.9] takes the form:

$$
\sigma=\int_{-\infty}^{+\infty} \kappa\left(\frac{\partial \varphi_{e q}(z)}{\partial z}\right)^{2} d z=\int_{0}^{1} \kappa \frac{\partial \varphi_{e q}}{\partial z} \partial \varphi_{e q}=\int_{0}^{1} \kappa \sqrt{\frac{2 \omega}{\kappa}} \varphi_{e q}\left(1-\varphi_{e q}\right) \partial \varphi_{e q}=\frac{1}{6} \sqrt{2 \omega \kappa}
$$

Furthermore, one can express the interface thickness as:

$$
\epsilon=\left[\max _{-\infty \leq z \leq+\infty}\left(\frac{\partial \varphi_{e q}(z)}{\partial z}\right)\right]^{-1}
$$

and, according to Eq. C.4 , one obtain:

$$
\epsilon=\left[\max _{-\infty \leq z \leq+\infty}\left(\frac{\partial \varphi_{e q}(z)}{\partial z}\right)\right]^{-1}=\left[\max _{\{0 \leq z \leq 1\}} \sqrt{\frac{2 \omega}{\kappa}}\left[\varphi_{e q}\left(1-\varphi_{e q}\right)\right]\right]^{-1}=4 \sqrt{\frac{\kappa}{2 \omega}}
$$

which enables to re-write Eq. C.8 as follows:

$$
\varphi_{e q}(z)=\frac{1}{2}\left[1+\tanh \left(\frac{2}{\epsilon} z\right)\right]
$$

At this stage, substituting Eq.C.12 in Eq.C.6 yields:

$$
\kappa=\frac{3}{2} \sigma \epsilon
$$

and the use of Eq.C.14 together with Eq. C.12 gives the depth of the double-well potential in the following form:

$$
\omega=12 \frac{\sigma}{\epsilon}
$$

Here, it is interesting to mention that all the relationships obtained in this appendix are equivalent to those given by [34] and [28] by setting $\epsilon=4 \delta$ in [34] and $\epsilon=2 \lambda$ with $\alpha=1$ in [28].

\section{Appendix D. Centered Finite Differences scheme}

In this appendix, we present the centered Finite Differences scheme used to solve one-dimensional problem. Before detailing the numerical scheme, let us define some practical notations: $\Omega$ denotes the non-uniformly discretized one-dimensional domain and $\Gamma$ its boundary; $\Delta t$ refers to the time increment between two successive time steps $t^{n}$ and $t^{n+1} ; N$ is the maximum number of grid points, $i$ the node index whereas $\Delta z_{i+1}$ and $\Delta z_{i}$ correspond to the space sizes from $i$ to $i+1$ and from $i$ to $i-1$, respectively; and the curly brackets define a set of variables indexed by $i$ for $i=0, \ldots, N$.

In the following, we assume that $\left\{\varphi_{i}^{n}\right\},\left\{T_{i}^{n}\right\},\left\{\left(x_{o}\right)_{i}^{n}\right\}$ are known at the time step $t^{n}$. In these conditions, by focusing on Eq. $49 a$, Eq. $49 b$ and Eq. $49 c$, the time marching reads: 
1. Using $\left\{\varphi_{i}^{n}\right\},\left\{T_{i}^{n}\right\},\left\{\left(x_{o}\right)_{i}^{n}\right\}$, perform all the equilibrium calculations and find an estimation of $\left\{\left(g^{l}\right)_{i}^{n+1}\right\}$, $\left\{\left(g^{s}\right)_{i}^{n+1}\right\},\left\{\left(x_{o}^{s}\right)_{i}^{n+1}\right\},\left\{\left(x_{o}^{l}\right)_{i}^{n+1}\right\},\left\{\left(\tilde{\mu}_{o}\right)_{i}^{n+1}\right\}$ and $\left\{\left(\tilde{\mu}_{u}\right)_{i}^{n+1}\right\}$

2. Afterwards, calculate the discrete CALPHAD term $\left\{\left(g^{l}\right)_{i}^{n+1}-\left(g^{s}\right)_{i}^{n+1}-\left(\left(x_{o}^{l}\right)_{i}^{n+1}-\left(x_{o}^{s}\right)_{i}^{n+1}\right)\left(\left(\tilde{\mu}_{o}\right)_{i}^{n+1}-\left(\tilde{\mu}_{u}\right)_{i}^{n+1}\right)\right\}$ and find $\left\{\varphi_{i}^{n+1}\right\}$ such that:

$$
\begin{aligned}
\frac{\varphi_{i}^{n+1}-\varphi_{i}^{n}}{\Delta t} & =-M_{\varphi}\left\{-\rho h^{\prime}\left(\varphi_{i}^{n+1}\right)\left[\left(g^{l}\right)_{i}^{n+1}-\left(g^{s}\right)_{i}^{n+1}-\left(\left(x_{o}^{l}\right)_{i}^{n+1}-\left(x_{o}^{s}\right)_{i}^{n+1}\right)\left(\left(\tilde{\mu}_{o}\right)_{i}^{n+1}-\left(\tilde{\mu}_{u}\right)_{i}^{n+1}\right)\right]_{i}^{n+1}\right. \\
& +24 \frac{\sigma}{\epsilon} \varphi_{i}^{n+1} \times\left(1-\varphi_{i}^{n+1}\right) \times\left(1-2 \varphi_{i}^{n+1}\right) \\
& -\frac{3}{2} \sigma \epsilon\left[\frac{2 \varphi_{i+1}^{n+1}}{\Delta z_{i+1}\left(\Delta z_{i}+\Delta z_{i+1}\right)}-\frac{2 \varphi_{i}^{n+1}}{\Delta z_{i} \Delta z_{i+1}}+\frac{2 \varphi_{i-1}^{n+1}}{\Delta z_{i}\left(\Delta z_{i}+\Delta z_{i+1}\right)}\right]
\end{aligned}
$$

Here-above, when the mesh is uniform (i.e. $\Delta z_{i}=\Delta z \forall i=0, \ldots, N$ ), the latter term of the right-hand-side of Eq. D.1 degenerates in the well-known 3-point stencil Laplacian:

$$
\frac{3}{2} \sigma \epsilon\left[\frac{\varphi_{i+1}^{n+1}-2 \varphi_{i}^{n+1}+\varphi_{i-1}^{n+1}}{\Delta z^{2}}\right]
$$

Finally, for this problem, by assuming that the right side $(i=N)$ of the domain remains solid, the following Neumann-Dirichlet boundary conditions are considered:

$$
\begin{aligned}
\text { (Dirichlet) } & \varphi_{N}^{n+1}=1 \quad \forall n \geq 0 \\
\text { (Neumann) } & \varphi_{-1}^{n+1}=\varphi_{1}^{n+1} \quad \forall n \geq 0
\end{aligned}
$$

where the subscript $\cdot_{-1}$ denotes a virtual grid point located at a distance $\Delta z_{0}=\Delta z_{1}$ from the zero grid point. This non-linear problem is solved by using a standard Newton method.

3. At this stage, using the estimations of $\left\{\varphi_{i}^{n+1}\right\},\left\{T_{i}^{n+1}\right\},\left\{\left(\tilde{\mu}_{o}\right)_{i}^{n+1}\right\}$ and $\left\{\left(\tilde{\mu}_{u}\right)_{i}^{n+1}\right\}$, find $\left\{\left(x_{o}\right)_{i}^{n+1}\right\}$ such that:

$$
\begin{aligned}
\frac{\left(x_{o}\right)_{i}^{n+1}-\left(x_{o}\right)_{i}^{n}}{\Delta t}= & 2\left\{\frac{M_{c}^{i+\frac{1}{2}} \Delta \tilde{\mu}_{i+1}^{n+1}}{\Delta z_{i+1}\left(\Delta z_{i}+\Delta z_{i+1}\right)}-\frac{\Delta \tilde{\mu}_{i}^{n+1}\left[\Delta z_{i} M_{c}^{i+\frac{1}{2}}+\Delta z_{i+1} M_{c}^{i-\frac{1}{2}}\right]}{\Delta z_{i} \Delta z_{i+1}\left(\Delta z_{i}+\Delta z_{i+1}\right)}+\frac{M_{c}^{i-\frac{1}{2}} \Delta \tilde{\mu}_{i-1}^{n+1}}{\Delta z_{i}\left(\Delta z_{i}+\Delta z_{i+1}\right)}\right. \\
& \left.+\frac{M_{T}^{i+\frac{1}{2}} \ln \left(T_{i+1}^{n+1}\right)}{\Delta z_{i+1}\left(\Delta z_{i}+\Delta z_{i+1}\right)}-\frac{\ln \left(T_{i}^{n+1}\right)\left[\Delta z_{i} M_{T}^{i+\frac{1}{2}}+\Delta z_{i+1} M_{T}^{i-\frac{1}{2}}\right]}{\Delta z_{i} \Delta z_{i+1}\left(\Delta z_{i}+\Delta z_{i+1}\right)}+\frac{M_{T}^{i-\frac{1}{2}} \ln \left(T_{i-1}^{n+1}\right)}{\Delta z_{i}\left(\Delta z_{i}+\Delta z_{i+1}\right)}\right\} \text { (D.4) }
\end{aligned}
$$

where $\Delta \tilde{\mu}_{i}^{n+1}=\left[\left(\tilde{\mu}_{o}\right)_{i}^{n+1}-\left(\tilde{\mu}_{u}\right)_{i}^{n+1}\right]$ for $i=0, \ldots, N$ whereas $M_{T}^{i-\frac{1}{2}}, M_{T}^{i+\frac{1}{2}}, M_{c}^{i-\frac{1}{2}}$ and $M_{c}^{i+\frac{1}{2}}$ are discrete mobility coefficients given by:

$$
M_{\alpha}^{i+\frac{j}{2}}=\frac{M_{\alpha}\left(\varphi_{i+j}^{n+1},\left(x_{o}\right)_{i+j}^{n}\right)+M_{\alpha}\left(\varphi_{i}^{n+1},\left(x_{o}\right)_{i}^{n}\right)}{2} \text { for } \alpha=T, C \text { and } j= \pm 1
$$

Here-again, when the mesh is uniform (i.e. $\Delta z_{i}=\Delta z \forall i=0, \ldots, N$ ), the right-hand-side of Eq. D.4] reduces to:

$$
\begin{aligned}
& \frac{M_{c}^{i+\frac{1}{2}} \Delta \tilde{\mu}_{i+1}^{n+1}-\left(M_{c}^{i+\frac{1}{2}}+M_{c}^{i-\frac{1}{2}}\right) \Delta \tilde{\mu}_{i}^{n+1}+M_{c}^{i-\frac{1}{2}} \Delta \tilde{\mu}_{i-1}^{n+1}}{\Delta z^{2}} \\
& +\frac{M_{T}^{i+\frac{1}{2}} \ln \left(T_{i+1}^{n+1}\right)-\left(M_{T}^{i+\frac{1}{2}}+M_{T}^{i-\frac{1}{2}}\right) \ln \left(T_{i}^{n+1}\right)+M_{T}^{i-\frac{1}{2}} \ln \left(T_{i-1}^{n+1}\right)}{\Delta z^{2}}
\end{aligned}
$$


Regarding to the boundary conditions associated to this discretization, we consider the following NeumannNeumann boundary conditions:

$$
\begin{aligned}
M_{c}^{i+\frac{1}{2}}\left(\Delta \tilde{\mu}_{i+1}^{n+1}-\Delta \tilde{\mu}_{i}^{n+1}\right)+M_{T}^{i+\frac{1}{2}}\left[\ln \left(T_{i+1}^{n+1}\right)-\ln \left(T_{i}^{n+1}\right)\right] & =M_{c}^{i-\frac{1}{2}}\left(\Delta \tilde{\mu}_{i-1}^{n+1}-\Delta \tilde{\mu}_{i}^{n+1}\right) \\
& +M_{T}^{i-\frac{1}{2}}\left[\ln \left(T_{i-1}^{n+1}\right)-\ln \left(T_{i}^{n+1}\right)\right] \text { for } i=0 \text { and } i=N
\end{aligned}
$$

where we have defined two virtual grid points located at a distance $\Delta z_{0}=\Delta z_{1}$ from the zero grid point and $\Delta z_{N}=\Delta z_{N-1}$ from the point number $N$, respectively.

\section{References}

[1] B. Lewis, W. Thompson, F. Iglesias, 2.20-fission product chemistry in oxide fuels, Comprehensive Nuclear Materials 2.

[2] C. Guéneau, N. Dupin, B. Sundman, C. Martial, J.-C. Dumas, S. Gossé, S. Chatain, F. De Bruycker, D. Manara, R. J. Konings, Thermodynamic modelling of advanced oxide and carbide nuclear fuels: Description of the u-pu-0-c systems, Journal of nuclear materials 419 (1-3) (2011) 145-167.

[3] M. Piro, J. Banfield, K. T. Clarno, S. Simunovic, T. M. Besmann, B. Lewis, W. Thompson, Coupled thermochemical, isotopic evolution and heat transfer simulations in highly irradiated uo2 nuclear fuel, Journal of Nuclear Materials 441 (1-3) (2013) 240-251.

[4] B. Baurens, J. Sercombe, C. Riglet-Martial, L. Desgranges, L. Trotignon, P. Maugis, 3d thermo-chemical-mechanical simulation of power ramps with alcyone fuel code, Journal of Nuclear Materials 452 (1-3) (2014) 578-594.

[5] P. Konarski, J. Sercombe, C. Riglet-Martial, L. Noirot, I. Zacharie-Aubrun, K. Hanifi, M. Fregonèsé, P. Chantrenne, 3d simulation of a power ramp including fuel thermochemistry and oxygen thermodiffusion, Journal of Nuclear Materials 519 (2019) 104-120.

[6] M. Piro, T. M. Besmann, S. Simunovic, B. Lewis, W. Thompson, Numerical verification of equilibrium thermodynamic computations in nuclear fuel performance codes, Journal of Nuclear Materials 414 (3) (2011) 399-407.

[7] H. Loukusa, T. Ikonen, V. Valtavirta, V. Tulkki, Thermochemical modeling of nuclear fuel and the effects of oxygen potential buffers, Journal of Nuclear Materials 481 (2016) 101-110.

[8] B. Sundman, X.-G. Lu, H. Ohtani, The implementation of an algorithm to calculate thermodynamic equilibria for multi-component systems with non-ideal phases in a free software, Computational Materials Science 101 (2015) 127-137.

[9] B. Sundman, U. R. Kattner, M. Palumbo, S. G. Fries, Opencalphad-a free thermodynamic software, Integrating Materials and Manufacturing Innovation 4 (1) (2015) 1.

10] S. Simunovic, T. M. Besmann, Coupling of thermochemistry solver thermochimica with moose/bison, ORNL/TM-2015/322, ORNL 25.

[11] S. Simunovic, T. M. Besmann, E. Moore, M. Poschmann, M. H. Piro, K. T. Clarno, J. W. McMurray, W. A. Wieselquist, Modeling and simulation of oxygen transport in high burnup lwr fuel, Journal of Nuclear Materials (2020) 152194.

[12] K. Samuelsson, J.-C. Dumas, B. Sundman, M. Lainet, An improved method to evaluate the "joint oxyde-gaine" formation in (u, pu) o2 irradiated fuels using the germinal v2 code coupled to calphad thermodynamic computations, EPJ Nuclear Sciences \& Technologies 6 (2020) 47.

[13] C. Introïni, J. Sercombe, B. Sundman, Development of a robust, accurate and efficient coupling between pleiades/alcyone 2.1 fuel performance code and the opencalphad thermo-chemical solver, Nuclear Engineering and Design 369 (2020) 110818.

[14] E. Cordfunke, R. Konings, Thermochemical data for reactor materials and fission products: The ecn database, Journal of phase equilibria 14 (4) (1993) 457-464.

[15] J. Hales, S. Novascone, B. Spencer, R. Williamson, G. Pastore, D. Perez, Verification of the bison fuel performance code, Annals of Nuclear Energy 71 (2014) 81-90.

[16] I. N. Laboratory, Bison: A finite element-based nuclear fuel performance code. https://bison.inl.gov (2020).

[17] T. M. Besmann, J. W. McMurray, S. Simunovic, Application of thermochemical modeling to assessment/evaluation of nuclear fuel behavior, Calphad 55 (2016) 47-51.

[18] C. W. Bale, E. Bélisle, P. Chartrand, S. Decterov, G. Eriksson, A. Gheribi, K. Hack, I.-H. Jung, Y.-B. Kang, J. Melançon, et al., Reprint of: Factsage thermochemical software and databases, 2010-2016, Calphad 55 (2016) 1-19.

[19] N. Moelans, B. Blanpain, P. Wollants, An introduction to phase-field modeling of microstructure evolution, Calphad 32 (2) (2008) $268-294$.

[20] D. Jacqmin, Calculation of two-phase navier-stokes flows using phase-field modeling, Journal of Computational Physics 155 (1) (1999) 96-127.

$535[21]$ F. Boyer, C. Lapuerta, Study of a three component cahn-hilliard flow model, ESAIM: Mathematical Modelling and Numerical AnalysisModélisation Mathématique et Analyse Numérique 40 (4) (2006) 653-687.

[22] F. Boyer, C. Lapuerta, S. Minjeaud, B. Piar, M. Quintard, Cahn-hilliard/navier-stokes model for the simulation of three-phase flows, Transport in Porous Media 82 (3) (2010) 463-483.

[23] C. Introïni, Interaction entre un fluide à haute température et un béton: contribution à la modélisation des échanges de masse et de chaleur, Ph.D. thesis (2010).

[24] Y. Lu, T. Helfer, B. Bary, O. Fandeur, An efficient and robust staggered algorithm applied to the quasi-static description of brittle fracture by a phase-field approach, Computer Methods in Applied Mechanics and Engineering 370 (2020) 113218.

[25] J. Tiaden, B. Nestler, H.-J. Diepers, I. Steinbach, The multiphase-field model with an integrated concept for modelling solute diffusion, Physica D: Nonlinear Phenomena 115 (1-2) (1998) 73-86.

[26] C. Cardon, R. Le Tellier, M. Plapp, Modelling of liquid phase segregation in the uranium-oxygen binary system, Calphad 52 (2016) 47-56.

[27] A. A. Wheeler, W. J. Boettinger, G. B. McFadden, Phase-field model for isothermal phase transitions in binary alloys, Physical Review A 45 (10) (1992) 7424. 
[28] S. G. Kim, W. T. Kim, T. Suzuki, Phase-field model for binary alloys, Physical review e 60 (6) (1999) 7186.

[29] V. Tiwari, A consistent approach for coupling lumped-parameter and phase-field models for in-vessel corium to thermodynamic databases., Ph.D. thesis, Université Paris-Saclay (ComUE) (2019).

[30] J. Hirschhorn, M. R. Tonks, A. Aitkaliyeva, C. Adkins, A study of constituent redistribution in u-zr fuels using quantitative phase-field modeling and sensitivity analysis, Journal of Nuclear Materials 523 (2019) 143-156.

[31] J. Hirschhorn, M. Tonks, A. Aitkaliyeva, C. Adkins, Reexamination of a u-zr diffusion couple experiment using quantitative phase-field modeling and sensitivity analysis, Journal of Nuclear Materials 529 (2020) 151929.

[32] M. J. Welland, Simulation of melting uranium dioxide nuclear fuel simulation de fusion de combustible nucléaire fait de bioxyde d'uranium, Ph.D. thesis, Royal Military College of Canada (2009).

[33] M. Welland, W. Thompson, B. Lewis, D. Manara, Computer simulations of non-congruent melting of hyperstoichiometric uranium dioxide, Journal of nuclear materials 385 (2) (2009) 358-363.

[34] M. Welland, B. Lewis, W. Thompson, Review of high temperature thermochemical properties and application in phase-field modelling of incipient melting in defective fuel, Journal of nuclear materials 412 (3) (2011) 342-349.

[35] E. Moore, C. Guéneau, J.-P. Crocombette, Diffusion model of the non-stoichiometric uranium dioxide, Journal of Solid State Chemistry 203 (2013) 145-153.

[36] E. Moore, C. Guéneau, J.-P. Crocombette, Oxygen diffusion model of the mixed (u, pu) o2 \pm x: Assessment and application, Journal of Nuclear Materials 485 (2017) 216-230.

[37] TAF-ID, https://www.oecd-nea.org/science/taf-id/.

[38] E. Geiger, C. Guéneau, Y. Pontillon, E. Corcoran, Modelling nuclear fuel behaviour with taf-id: Calculations on the verdon-1 experiment, representative of a nuclear severe accident, Journal of Nuclear Materials 522 (2019) 294-310.

[39] C. Guéneau, N. Dupin, L. Kjellqvist, E. Geiger, M. Kurata, S. Gossé, E. Corcoran, A. Quaini, R. Hania, A. Smith, et al., Taf-id: An international thermodynamic database for nuclear fuels applications, Calphad 72102212.

40] C. Introïni, J. Sercombe, C. Guéneau, P. Goldbronn, Pleiades alcyone 3.5d simulation of a power ramp including opencalphad fuel thermochemistry with taf-id, in: NuFuel MMSNF workshop, 2019.

[41] H. Lukas, S. G. Fries, B. Sundman, Computational thermodynamics: the Calphad method, Cambridge university press, 2007.

[42] M. Hillert, B. Jansson, B. Sundman, et al., A two-sublattice model for molten solutions with different tendency for ionization, Metallurgical Transactions A 16 (1) (1985) 261-266.

43] Y. A. Coutinho, N. Vervliet, L. De Lathauwer, N. Moelans, Combining thermodynamics with tensor completion techniques to enable multicomponent microstructure prediction, npj Computational Materials 6 (1) (2020) 1-11.

[44] S. R. De Groot, P. Mazur, Non-equilibrium thermodynamics, Courier Corporation, 2013.

[45] A microscopic theory for antiphase boundary motion and its application to antiphase domain coarsening Acta Metallurgica 27 (6) (1979) 1085-1095. doi:https://doi.org/10.1016/0001-6160(79)90196-2 URL https://www.sciencedirect.com/science/article/pii/0001616079901962

[46] M. J. Welland, D. Wolf, J. E. Guyer, Multicomponent phase-field model for extremely large partition coefficients, Physical Review E 89 (1) (2014) 012409.

[47] L. Onsager, Reciprocal relations in irreversible processes. i., Physical review 37 (4) (1931) 405.

[48] M. J. Welland, 2.08 - matter transport in fast reactor fuels in: R. J. Konings, R. E. Stoller (Eds.), Comprehensive Nuclear Materials (Second Edition), second edition Edition, Elsevier, Oxford, 2020, pp. 200-247. doi :https://doi .org/10.1016/B978-0-12-803581-8. $10304-2$ URLhttps://www.sciencedirect.com/science/article/pii/B9780128035818103042

[49] R. E. Howard, A. B. Lidiard, Matter transport in solids Reports on Progress in Physics 27 (1) (1964) 161-240. doi : 10.1088/0034-4885/ $27 / 1 / 305$ URL https://doi.org/10.1088/0034-4885/27/1/305

[50] S. R. de Groot, Sur la thermodynamique de quelques processus irréversibles. ii. diffusion thermique et phénomènes connexes.

[51] E. Dougherty Jr, H. Drickamer, A theory of thermal diffusion in liquids, The Journal of Chemical Physics 23 (2) (1955) 295-309.

[52] C. Introïni, M. Quintard, F. Duval, Cahn-hilliard modelling for a two-phase compositional system, in: Proceedings of 6th International Conference on Computational Heat and Mass Transfer, Guangzhou, 2009.

53] C. Takoukam-Takoundjou, E. Bourasseau, M. J. D. Rushton, V. Lachet, Optimization of a new interatomic potential to investigate the thermodynamic properties of hypo-stoichiometric mixed oxide fuel ul-ypuyo2-x, Journal of Physics Condensed Matter 32 (50).

[54] C. Takoukam-Takoundjou, E. Bourasseau, V. Lachet, Study of thermodynamic properties of ul-ypuyo2 mox fuel using classical molecular monte carlo simulations, Journal of Nuclear Materials 534 (152125).

[55] D. Bathellier, M. Lainet, M. Freyss, P. Olsson, E. Bourasseau, A new heat capacity law for uo2, puo2 and (u,pu)o2 derived from molecular dynamics simulations and useable in fuel performance codes Journal of Nuclear Materials 549 (2021) 152877. doi : https://doi . org/ $10.1016 / j \cdot$ jnucmat.2021.152877 URLhttps://www.sciencedirect.com/science/article/pii/S0022311521001008

[56] G. Alefeld, F. A. Potra, Y. Shi, Algorithm 748: enclosing zeros of continuous functions, ACM Transactions on Mathematical Software (TOMS) 21 (3) (1995) 327-344.

[57] B. Michel, I. Ramière, I. Viallard, C. Introini, M. Lainet, N. Chauvin, V. Marelle, A. Boulore, T. Helfer, R. Masson, J. Sercombe, J. Dumas, L. Noirot, S. Bernaud, Chapter 9 - two fuel performance codes of the pleiades platform: Alcyone and germinal in: J. Wang, X. Li, C. Allison, J. Hohorst (Eds.), Nuclear Power Plant Design and Analysis Codes, Woodhead Publishing Series in Energy, Woodhead Publishing, 2021, pp. 207-233. doi : https://doi .org/10.1016/B978-0-12-818190-4.00009-7. URL https://www.sciencedirect.com/science/article/pii/B9780128181904000097

[58] P. Konarski, Thermo-chemical-mechanical modeling of nuclear fuel behavior: Impact of oxygen transport in the fuel on pellet cladding interaction, Ph.D. thesis, Université de Lyon (2019).

[59] M. Plapp, Unified derivation of phase-field models for alloy solidification from a grand-potential functional, Physical Review E 84 (3) 
(2011) 031601.

[60] M. Welland, E. Tenuta, A. Prudil, Linearization-based method for solving a multicomponent diffusion phase-field model with arbitrary solution thermodynamics, Physical Review E 95 (6) (2017) 063312.

[61] L. K. Aagesen, Y. Gao, D. Schwen, K. Ahmed, Grand-potential-based phase-field model for multiple phases, grains, and chemical components, Physical Review E 98 (2) (2018) 023309. 